The Alaska Volcano Observatory is a cooperative program of the U.S. Geological Survey, University of Alaska Fairbanks Geophysical Institute, and the Alaska Division of Geological and Geophysical Surveys. The Alaska Volcano Observatory is funded by the U.S. Geological Survey Volcano Hazards Program and the State of Alaska.

\title{
2011 Volcanic Activity in Alaska: Summary of Events and Response of the Alaska Volcano Observatory
}

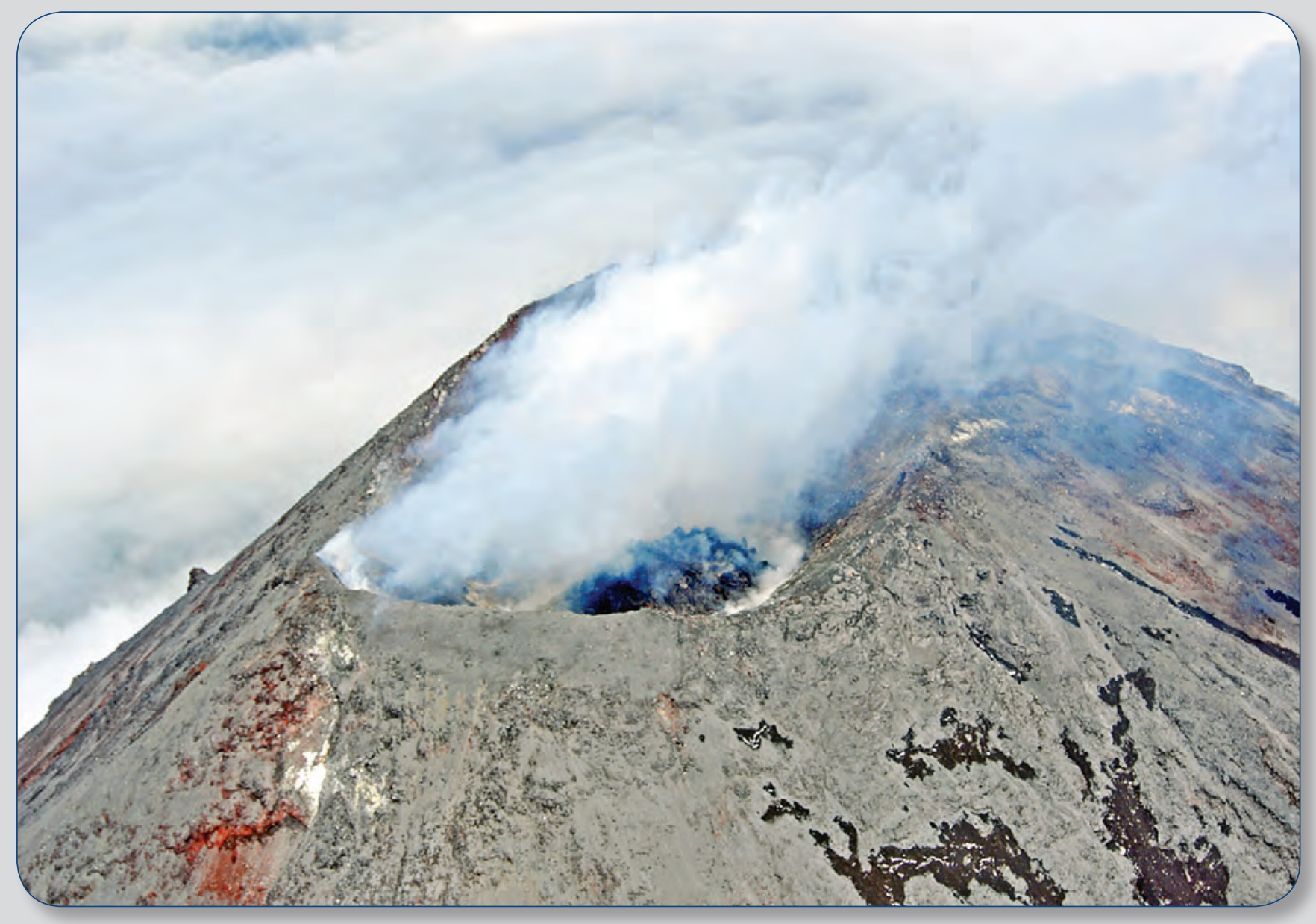

Scientific Investigations Report 2014-5159 
Cover: Aerial photograph of the August 2011 lava dome in the summit crater of Cleveland volcano. The lava dome is about $60 \mathrm{~m}(200 \mathrm{ft})$ in diameter in a 200 -m-wide (660 ft) crater, and was extruded sometime between July 7 and August 8, 2011.

Photograph courtesy of Dave Withrow, National Oceanic and Atmospheric

Administration, taken on August 8, 2011. AV0 database image at URL: http://www.avo. alaska.edu/images/image. .php?id=36312. 


\section{Volcanic Activity in Alaska: Summary of Events and Response of the Alaska Volcano Observatory}

By Robert G. McGimsey, J. Zebulon Maharrey, and Christina A. Neal

The Alaska Volcano Observatory is a cooperative program of the U.S. Geological Survey, University of Alaska Fairbanks Geophysical Institute, and the Alaska Division of Geological and Geophysical Surveys. The Alaska Volcano Observatory is funded by the U.S. Geological Survey Volcano Hazards Program and the State of Alaska.

Scientific Investigations Report 2014-5159 


\title{
U.S. Department of the Interior SALLY JEWELL, Secretary
}

\section{U.S. Geological Survey Suzette M. Kimball, Acting Director}

\author{
U.S. Geological Survey, Reston, Virginia: 2014
}

For more information on the USGS - the Federal source for science about the Earth, its natural and living resources, natural hazards, and the environment, visit http://www.usgs.gov or call 1-888-ASK-USGS

For an overview of USGS information products, including maps, imagery, and publications, visit http://www.usgs.gov/pubprod

To order this and other USGS information products, visit http://store.usgs.gov

Any use of trade, firm, or product names is for descriptive purposes only and does not imply endorsement by the U.S. Government.

Although this information product, for the most part, is in the public domain, it also may contain copyrighted materials as noted in the text. Permission to reproduce copyrighted items must be secured from the copyright owner.

Suggested citation:

McGimsey, R.G., Maharrey, J.Z., and Neal, C.A., 2014, 2011 Volcanic activity in Alaska-Summary of events and response of the Alaska Volcano Observatory: U.S. Geological Survey Scientific Investigations Report 2014-5159, 50 p., http://dx.doi.org/10.3133/sir20145159.

ISSN 2328-0328 (online) 


\section{Contents}

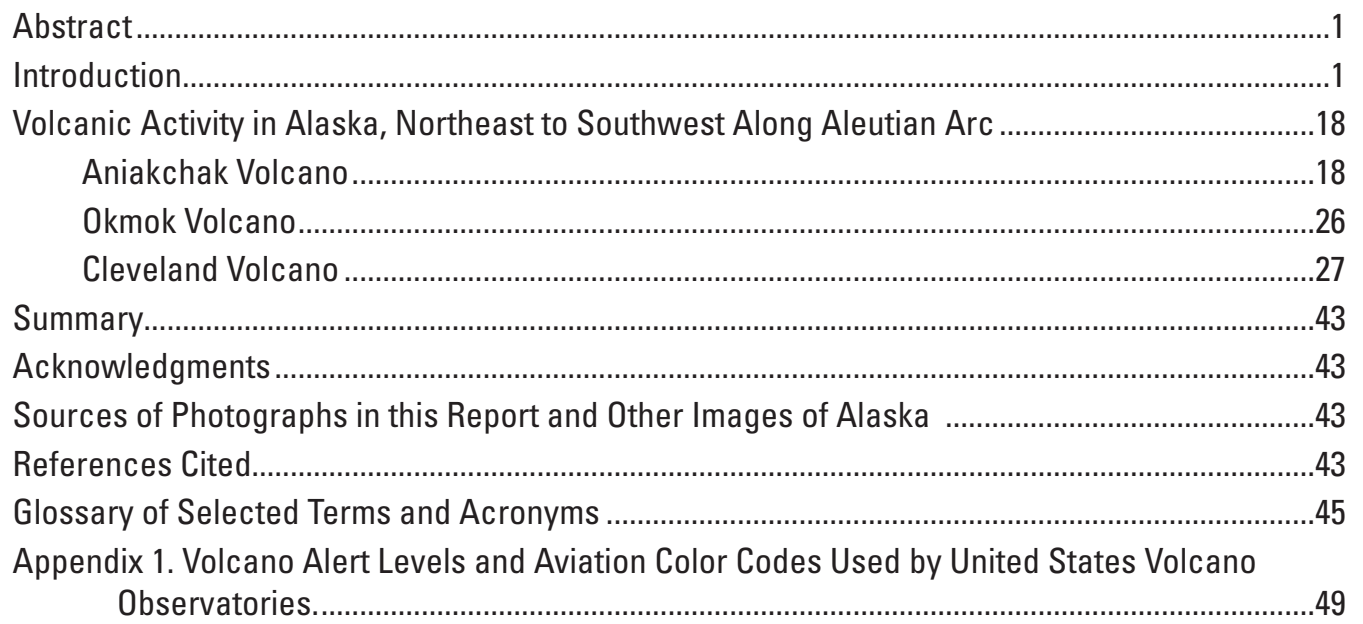




\section{Figures}

1. Map showing historically active volcanoes in Alaska along with place names used in this report... .2

2. Oblique Google Earth ${ }^{\mathrm{TM}}$ view of Aniakchak caldera looking to the north.. 19

3. Photograph showing larger of two maar craters on the southeastern floor of Aniakchak caldera as viewed from the southeastern caldera rim .20

4. Photograph of the northwestern rim of the maar 21

5. Photographs of drainage between the maar and the Aniakchak River before and after the flood event showing displacement of the stream channel and erosion of the flood plain and fan ...

6. Comparative aerial oblique views east showing the Aniakchak River exiting the

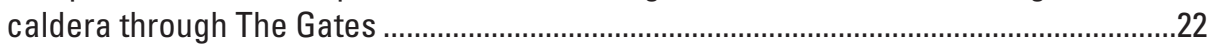

7. Comparative images of the large maar in Aniakchak caldera..........................................23

8. View south of the breach in the northern rim of the maar crater lake on the eastern

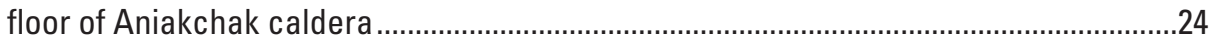

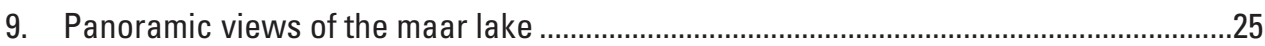

10. Elevated temperature anomaly at the summit of Cleveland volcano in a NOAA Advanced Very High Resolution Radiometer (AVHRR) satellite image, channel 3 (thermal band) from 06:12 UTC July 17, 2011 ..............................................................28

11. MODIS satellite thermal image of the Islands of the Four Mountains and Umnak

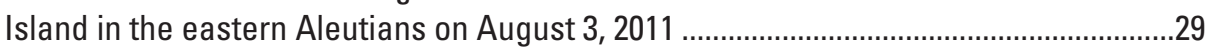

12. Landsat images of Cleveland volcano from August 7, 2011........................................30

13. WorldView-1 satellite image of the summit of Cleveland volcano, August 9, 2011 .........32

14. Oblique aerial photographs of the summit crater of Cleveland volcano on August 8 , 2011

15. Time sequence of TerraSAR-X images of the Cleveland cone and summit crater

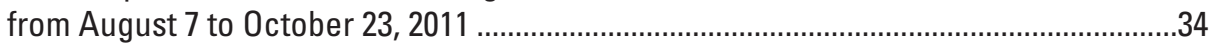

16. NOAA AVHRR satellite image from 14:02 UTC on December 29, 2011 ..........................35

17. Record of the December 29, 2011, explosion at Cleveland volcano on seismic stations OKWE, OKRE, and OKER. 36

\section{Tables}

1. History of seismic monitoring of Alaskan volcanoes from August 1971 through December 2011.

2. Summary of 2011 volcanic activity in Alaska, including actual eruptions, possible eruptions, unusual increases in seismicity or fumarolic activity.

3. Alaskan volcanoes with Aviation Color Code and Volcano Alert Level changes in $\mathbf{2 0 1 1 . 5}$

4a. Compilation by year of volcanoes included in an Alaska Volcano Observatory Annual Summary, 1992-2011.

4b. Compilation by volcano for particular years included in an Alaska Volcano Observatory Annual Summary, 1992-2011. . .10

4c. Citations for Alaska Volcano Observatory Annual Summary reports, 1992-2011. 16 


\section{Conversion Factors and Datum}

\section{Conversion Factors}

Inch-Pound to SI

\begin{tabular}{lcl}
\hline \multicolumn{1}{c}{ Multiply } & \multicolumn{1}{c}{ By } & \multicolumn{1}{c}{ To obtain } \\
\hline acre & 4,047 & square meter $\left(\mathrm{m}^{2}\right)$ \\
cubic mile $\left(\mathrm{mi}^{3}\right)$ & 4.168 & cubic kilometer $\left(\mathrm{km}^{3}\right)$ \\
foot (ft) & 0.000305 & kilometer $(\mathrm{km})$ \\
foot (ft) & 0.3048 & meter $(\mathrm{m})$ \\
inch (in.) & 2.54 & centimeter $(\mathrm{cm})$ \\
inch (in.) & 25.4 & millimeter $(\mathrm{mm})$ \\
mile (mi) & 1.609 & kilometer $(\mathrm{km})$ \\
ton per day (ton/d) & 0.9072 & metric ton per day \\
\hline
\end{tabular}

Temperature in degrees Fahrenheit $\left({ }^{\circ} \mathrm{F}\right)$ may be converted to degrees Celsius $\left({ }^{\circ} \mathrm{C}\right)$ as follows:

$$
{ }^{\circ} \mathrm{C}=\left({ }^{\circ} \mathrm{F}-32\right) / 1.8
$$

SI to Inch-Pound

\begin{tabular}{lcl}
\hline \multicolumn{1}{c}{ Multiply } & \multicolumn{1}{c}{ By } & \multicolumn{1}{c}{ To obtain } \\
\hline centimeter $(\mathrm{cm})$ & 0.3937 & inches (in.) \\
millimeter $(\mathrm{mm})$ & 0.03937 & inch (in.) \\
meter $(\mathrm{m})$ & 3.281 & foot (ft) \\
kilometer $(\mathrm{km})$ & 0.6214 & mile (mi) \\
kilometer $(\mathrm{km})$ & 0.5400 & mile, nautical (nmi) \\
kilometer $(\mathrm{km})$ & 3,281 & foot (ft) \\
square meter $\left(\mathrm{m}^{2}\right)$ & 0.0002471 & acre \\
cubic meter $\left(\mathrm{m}^{3}\right)$ & 1.308 & cubic yard $\left(\mathrm{yd}^{3}\right)$ \\
cubic kilometer $\left(\mathrm{km}^{3}\right)$ & 0.2399 & cubic mile $\left(\mathrm{mi}^{3}\right)$ \\
metric ton per day & 1.1022 & ton per day (ton/d) \\
\hline
\end{tabular}

Temperature in degrees Celsius $\left({ }^{\circ} \mathrm{C}\right)$ may be converted to degrees Fahrenheit $\left({ }^{\circ} \mathrm{F}\right)$ as follows:

$$
{ }^{\circ} \mathrm{F}=\left(1.8 \times^{\circ} \mathrm{C}\right)+32 .
$$

\section{Datum}

Altitude and elevation as used in this report, refers to distance above sea level, unless otherwise noted. 



\title{
2011 Volcanic Activity in Alaska: Summary of Events and Response of the Alaska Volcano Observatory
}

\author{
By Robert G. McGimsey, ${ }^{1}$ J. Zebulon Maharrey ${ }^{2}$, and Christina A. Neal'
}

\section{Abstract}

The Alaska Volcano Observatory (AVO) responded to eruptions, possible eruptions, and volcanic unrest at or near three separate volcanic centers in Alaska during 2011. The year was highlighted by the unrest and eruption of Cleveland Volcano in the central Aleutian Islands. AVO annual summaries no longer report on activity at Russian volcanoes.

\section{Introduction}

The Alaska Volcano Observatory (AVO) monitors, reports, and studies volcanic unrest at Alaskan volcanoes. The year 2011 was dominated by the eruption of Cleveland volcano in the central Aleutian Islands. AVO also responded to new and ongoing volcanic unrest at two other volcanoes in Alaska, Aniakchak, and Okmok volcanoes (fig. 1).

Of the 52 historically active volcanoes in Alaska (Schaefer and others, 2014), 33 were monitored in 2011 with a network of seismometers sufficiently reliable in their operation to consistently track earthquake activity (fig. 1; table 1) (Dixon and others, 2012). Korovin was delisted (considered not seismically monitored) in October 2011 because of low signal-to-noise problems with two of the four stations in the seismic network (J. Dixon, USGS, written commun., 2013), reducing the number of seismically monitored volcanoes to 32. Seismic stations are in place at two additional volcanoes (Little Sitkin and Semisopochnoi, fig. 1); however, telemetry links are intermittent and background seismicity has not been confidently determined. Thus, AVO does not yet consider these volcanoes formally monitored with seismic instrumentation. AVO's routine monitoring program in 2011 included twicedaily analysis of seismicity and satellite imagery, web cameras, occasional overflight observations, airborne-gas measurements, and a compilation of pilot reports and observations from local residents and mariners. Additionally, AVO receives real-time deformation information from permanent Global Positioning System (GPS) stations at four Alaskan volcanoes (Okmok, Augustine, Akutan, and Mount Spurr). In recent years, periodic analysis of Interferometric Synthetic Aperture Radar (InSAR) imagery also has been used to detect deformation at volcanoes in Alaska (Lu and others, 2003, 2007; Lu, 2007).

AVO continues to participate by formal agreement with the Kamchatkan Volcanic Eruption Response Team (KVERT) and the Sakhalin Volcanic Eruption Response Team (SVERT) to aid in satellite monitoring of Russian volcanoes and support dissemination of hazard information (Neal and others, 2009). As of 2011, AVO will no longer report on Russian volcanoes in the Annual Summary; however, the Observatory continues to assist in broadcasting alerts about eruptive activity at Russian volcanoes in Kamchatka and the Central Kuriles. Readers interested in following the activity of Russian volcanoes are referred to the web sites of the Kamchatka and Sakhalin Volcanic Eruption Response Teams (http://www. kscnet.ru/ivs/kvert/index eng.php and http://www.imgg. $\underline{\mathrm{ru} / \text { ? id } \mathrm{d}=659}$ ) and to the Smithsonian Institution Global Volcanism Network (GVN) volcano database available at URL: http://www.volcano.si.edu/.

This report summarizes volcanic activity in Alaska in 2011 and briefly describes AVO's operational response. Descriptions are presented in geographic order from northeast to southwest along the Aleutian Arc. Each event summary ends with a paragraph providing background comments on the volcano in question. Information is derived primarily from AVO daily status reports, weekly updates and special information releases/statements, AVO email and internal electronic logs, and the Global Volcanism Program Bulletins. Table 1 is a history of seismic monitoring of Alaskan volcanoes from August 1971 through December 2011.

\footnotetext{
${ }^{1}$ U.S. Geological Survey, Alaska Volcano Observatory, 4230 University Dr., Anchorage, Alaska 99508-4664.

${ }^{2}$ University of Alaska-Fairbanks, Department of Geology and Geophysics, Geophysical Institute, 903 Koyukuk Drive, Fairbanks, Alaska 99775-7320.
} 


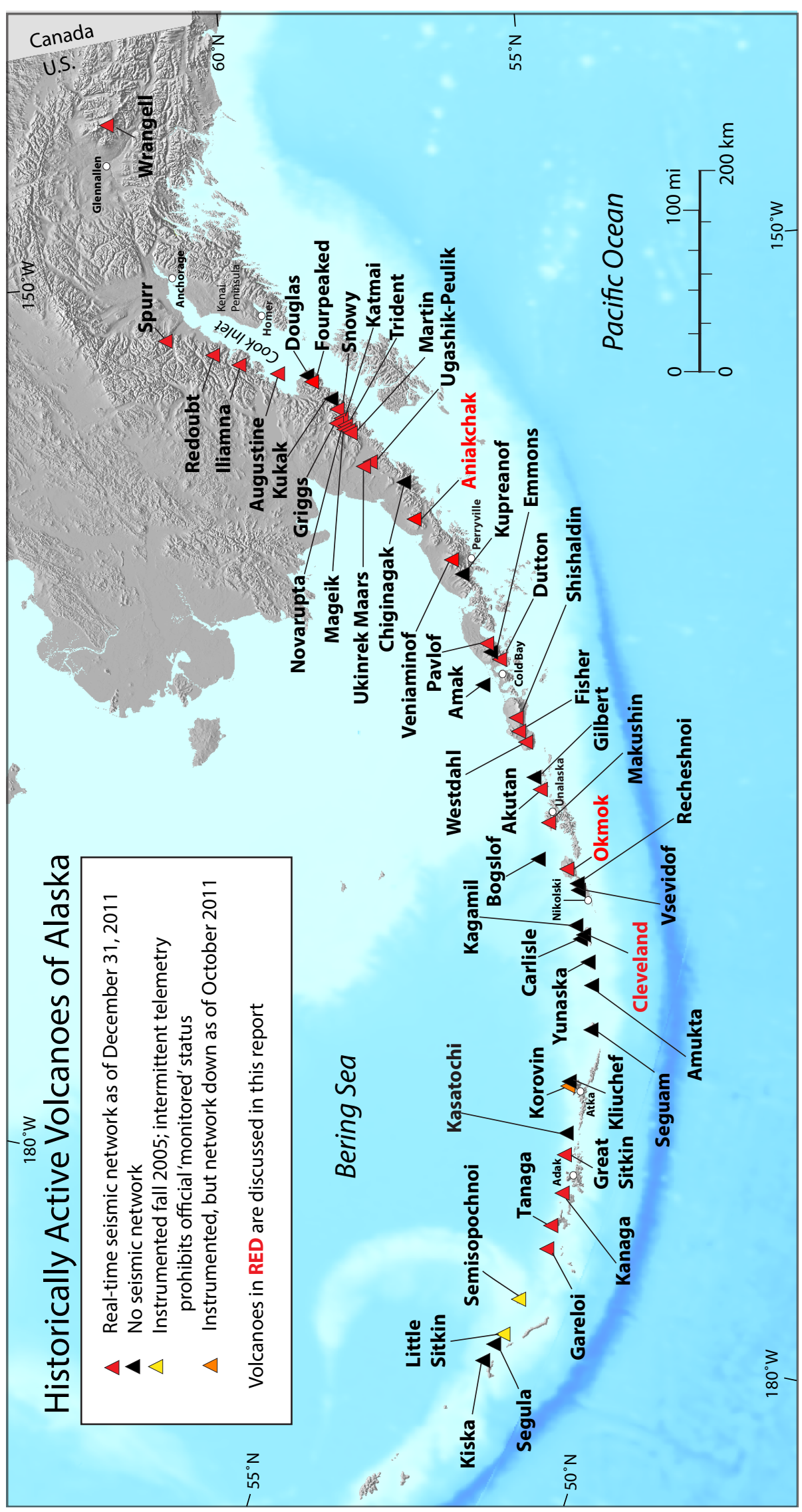

प

更

훙

늘

क̄ 흥

후일

d

응

등 ত

을 흥

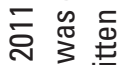

$=\frac{2}{2}$

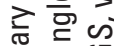

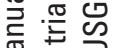

空

든 뜬 흘

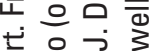

응

능 i

里主管

응 을

के $\frac{w}{\infty}$ क

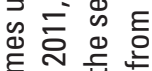

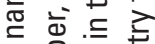

d 응

증응 음

焉

安言

듬

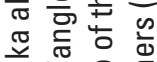

兽要号

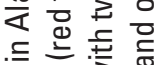

튼

む ळ के

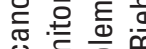

응 흐응 흥

충

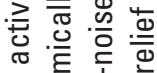

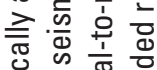

는 कs

迹㐘

品守

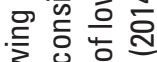

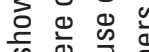

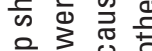

更 00

. 등

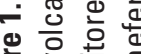

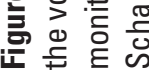


Table 1. History of seismic monitoring of Alaskan volcanoes from August 1971 through December 2011.

[History of seismic monitoring compiled by J. Dixon. "First station installed" refers to the date when AVO first received real-time data from the station. This date can be many months following initial fieldwork at the volcano. Alaska Volcano Observatory (AVO) considers the seismic network "complete" following installation and data transmission from a minimum of four seismic stations. Typically, AVO seismologists monitor the seismicity at the volcanic center for at least six months to understand background rates of seismicity before formally declaring a volcano seismically monitored and adding it to the monitored list. We note here the first mention of the seismic status of each monitored volcano in the AVO weekly update. Regularly issued written information statements began during the Redoubt eruption in 1989-90 and were expanded to include all Cook Inlet volcanoes in April 1991. The magnitude of completeness is the lowest magnitude earthquake that can confidently be located for activity at the volcanic center with an operational seismograph network. For more information on specific seismic network histories, readers are referred to the series of annual seismic summaries prepared by AVO (for example, Dixon and others, 2012)]

\section{Volcano}

Approximate start date of seismic monitoring
Magnitude of completeness

Wrangell
Spurr
Redoubt
Iliamna
Augustine
Fourpeaked
Katmai-South
(Martin, Mageik)
Katmai-North (Snowy)
Novarupta, Trident)

Ukinrek Maars/ Peulik

Aniakchak

Veniaminof

Pavlof

Dutton
First station installed - July 2000

Network complete - August 2001

Added to monitored list in weekly update - November 2001

First station installed - August 1971

Network complete - August 1989

Added to monitored list in weekly update - April 1991

First station installed - August 1971

Network complete - August 1988

Added to monitored list in weekly update - April 1991

First station installed - September 1987

Network complete (Min 4 stations) - September 1994

Added to monitored list in weekly update - April 1991

First station installed - October 1976

Network complete - August 1978

Added to monitored list in weekly update - April 1991

First station installed - September 2006

Network complete (Min 4 stations) - October 2006

Added to monitored list in weekly update - October 2006

First station installed - August 1988

Network complete - October 1998

Added to monitored list in weekly update - December 1998

First station installed - August 1988

Network complete (Min 4 stations) - July 1991

Added to monitored list in weekly update - November 1996

First station installed - August 1988

Network complete - July 1996

Added to monitored list in weekly update - November 1996

First station installed - March 2005

Network complete (Min 4 stations) - March 2005

Added to monitored list in weekly update - April 2005

First station installed - July 1997

Network complete - July $\mathbf{1 9 9 7}$

Added to monitored list in weekly update - November 1997

First station installed - February 2002

Network complete - February 2002

Added to monitored list in weekly update - September 2002

First station installed - July 1996

Network complete - July 1996

Added to monitored list in weekly update - November 1996

First station installed - July 1988

0.9
2

4 
Table 1. History of seismic monitoring of Alaskan volcanoes from August 1971 through December 2011.—Continued

[History of seismic monitoring compiled by J. Dixon. "First station installed" refers to the date when AVO first received real-time data from the station. This date can be many months following initial fieldwork at the volcano. Alaska Volcano Observatory (AVO) considers the seismic network "complete" following installation and data transmission from a minimum of four seismic stations. Typically, AVO seismologists monitor the seismicity at the volcanic center for at least six months to understand background rates of seismicity before formally declaring a volcano seismically monitored and adding it to the monitored list. We note here the first mention of the seismic status of each monitored volcano in the AVO weekly update. Regularly issued written information statements began during the Redoubt eruption in 1989-90 and were expanded to include all Cook Inlet volcanoes in April 1991. The magnitude of completeness is the lowest magnitude earthquake that can confidently be located for activity at the volcanic center with an operational seismograph network. For more information on specific seismic network histories, readers are referred to the series of annual seismic summaries prepared by AVO (for example, Dixon and others, 2012)]

Volcano

Approximate start date of seismic monitoring

Shishaldin (and Isantoski)

Westdahl (and Fisher)

Akutan

Makushin

Okmok

Korovin

Great Sitkin

Kanaga

Tanaga

Gareloi

Semisopochnoi (Cerberus)

Little Sitkin
First station installed - July 1997

Network complete - July 1997

Shishaldin added to list in weekly update - November 1997

Isantoski added to list in weekly update - December 1998

First station installed - August 1998

Network complete - October 1998

Added to monitored list in weekly update - December 1998

First station installed - March 1996

Network complete - July 1996

Added to monitored list in weekly update - November 1996

First station installed - July 1996

Network complete - July 1996

Added to monitored list in weekly update - November 1996

First station installed - January 2003

Network complete - January 2003

Added to monitored list in weekly update - January 2004

First station installed - July 2004

Network complete - July 2004

Added to monitored list in weekly update - December 2005

First station installed - September 1999

Network complete - September 1999

Added to monitored list in weekly update - December 1999

First station installed - September 1999

Network complete - September 1999

Added to monitored list in weekly update - December 2000

First station installed - August 2003

Network complete - August 2003

Added to monitored list in weekly update - June 2004

First station installed - August 2003

Network complete - September 2003

Added to monitored list in weekly update - June 2004

First station installed - September 2005

Network complete - September 2005

Added to monitored list in weekly update - not yet added

First station installed - September 2005

Added to monitored list in weekly update - not yet added 
Table 2 summarizes 2011 volcanic activity in Alaska. Table 3 summarizes changes in Aviation Color Codes in 2011 for Alaskan volcanoes. Table 4 presents cross-referenced lists of volcanic activity by year and by volcano for all previous reports (1992-2010).

Only activity that resulted in a significant investment of staff time (defined here as several hours or more for reaction, tracking, and follow-up) is included. Where more extensive published documentation for an episode of unrest exists, we provide key references. Over the course of the year, AVO typically receives dozens of reports of steaming, unusual cloud sightings, or false eruption reports. Most of these are resolved very quickly and are not tabulated here as part of the response record.

On rare occasions, AVO issues an information statement to dispel rumors of volcanic activity. In years past, the phrase "suspect volcanic activity" (SVA) has been used to characterize unusual activity that has subsequently been determined to be normal, or merely enhanced fumarolic activity, weather-related phenomena, or other non-volcanic events. Beginning with the 2006-year report, use of this term has ceased as it has presented us with problems of consistency.

This annual summary departs from including information about Russian volcanoes, a practice started in 1997 to recognize the cooperative relationship between $\mathrm{AVO}$ and KVERT. Beginning in 2005, information on the Kurile volcanoes also was included because of an additional collaboration with SVERT. We are ending this practice to acknowledge the success of both KVERT and SVERT in their reliable reporting on volcanic unrest and eruptions. As of 2011, $\mathrm{AVO}$ is no longer a formal partner with KVERT; however, we remain strong collaborators and continue to maintain situational awareness regarding eruptions in Kamchatka.

Table 2. Summary of 2011 volcanic activity in Alaska, including actual eruptions, possible eruptions, unusual increases in seismicity or fumarolic activity.

[Location of volcanoes shown in figure 1]

\begin{tabular}{|c|c|c|}
\hline Volcano & Date of activity & Type of activity \\
\hline Aniakchak & $\begin{array}{l}\text { Between late July } 2010 \text { and April 2011; } \\
\text { probably in August or September } 2010 \\
\text { based on local observations of heavy } \\
\text { precipitation. }\end{array}$ & $\begin{array}{l}\text { Catastrophic flood from the larger of two maars located on the eastern } \\
\text { floor of the caldera. }\end{array}$ \\
\hline
\end{tabular}

Table 3. Alaskan volcanoes with Aviation Color Code and Volcano Alert Level changes in 2011.

[Description of Aviation Color Codes is shown in appendix 1. Local times are only shown where color code changes were short-lived during rapidly evolving events. Volcanoes that do not have a real-time seismic network are not assigned a color code GREEN because without seismic data, Alaska Volcano Observatory has no definitive information that the level of activity at the volcano is at background. For these volcanoes, AVO uses the designation UNASSIGNED]

\begin{tabular}{|l|l|}
\hline \multicolumn{1}{|c|}{ Color Code } \\
\hline \multicolumn{2}{c|}{ CLEVELAND } \\
\hline YELLOW/ADVISORY & January 1-March 31 \\
\hline UNASSIGNED & March 31-July 20 \\
\hline YELLOW/ADVISORY & July 20-August 2 \\
\hline ORANGE/WATCH & August 2-August 30 \\
\hline YELLOW/ADVISORY & August 30-September 6 \\
\hline ORANGE/WATCH & September 6-November 3 \\
\hline YELLOW/ADVISORY & November 3-December 29 \\
\hline ORANGE/WATCH & December 29 (07:55 UTC)-December 30 (13:57 UTC) \\
\hline YELLOW/ADVISORY & December 30 (13:57 UTC)-December 31 \\
\hline
\end{tabular}


During 2011, our Remote Sensing program continued to evaluate Russian activity as part of its daily reporting, which was shared with our Russian colleagues. AVO also continues to alert U.S. and Canadian authorities when KVERT changes Aviation Color Code or during other significant activity that is likely to impact U.S. airspace or international airways. In 2011, AVO remained a formal partner in SVERT.

Altitudes and elevations reported are in feet and/or meters above sea level (ASL) unless otherwise noted, and time is reported as Alaska Standard Time (AKST) or Alaska Daylight Time (AKDT), as needed, with Coordinated Universal Time (UTC) in parentheses. For most satellite or geophysical instrumentation references, times are given in UTC. We preserve English or Inch-Pound units of measurement especially where they reflect the primary observations of distance or altitude such as those commonly received via pilot reports and aviation authorities in the United States. Elsewhere, measurements are presented in International System of Units (SI) with Inch-Pound Units in parentheses for convenience. Volcano locations in latitude and longitude and summit elevations are taken from the Alaska Volcano Observatory database and may differ slightly from previously published compilations.

\section{Increasing monitoring, increasing information, more to report}

As AVO has expanded instrumental monitoring and made use of the increasing number of high-resolution satellite platforms, the threshold of detection of volcanic unrest in Alaska has lowered considerably. In addition, increasing air and marine-vessel traffic in the Aleutians, along with improved Internet and other telecommunications infrastructure in remote Alaska, and the highly visible web presence of AVO may contribute to the increased number of reports of volcanic activity we receive, evaluate, and log. The focus of this report is on volcanic activity that represents a significant departure from 'background,' a somewhat loosely defined state of quiet at a given volcano. For a more quantitative picture of the level of volcanic unrest, readers are referred to the catalog of seismicity at Alaskan volcanoes, also produced on an annual basis (for example, Dixon and others, 2012).

\section{What is an "eruption"?}

The specific use of the term 'eruption' varies from scientist to scientist and there is no universally agreed-upon definition. Here, we adopt usage of the Smithsonian Institution's Global Volcanism Program, which defines eruptions as, “...events that involve explosive ejections of fragmental material, the effusion of liquid lava, or both. This fragmental material may be old as well as new; the explosive interaction of volcanically generated heat and near-surface water can cause dramatic eruptions without any fresh volcanic material reaching the surface..." (http://www.volcano.si.edu/faq.cfm\#q2). The element of this definition we wish to emphasize are the verbs 'eject' and 'effuse' which refer to dynamic surface processes that pose some level of hazard. The presence or absence of often ambiguous 'juvenile material' or fresh magma is not relevant to this use of the term eruption, particularly when communicating a potential hazard, which makes no distinction between juvenile and non-juvenile eruption products. This definition would not, however, include passive volcanic degassing or hydrothermalfluid discharge unless accidental solid fragments are entrained.

\section{What is an "historically active volcano"?}

AVO defines an "active" volcano as a volcanic center that has had an eruption (see above) or period of intense seismic or fumarolic activity that is inferred to reflect magma at shallow levels within the volcano. The "historic" period in Alaska is considered to be post mid-1700s when written records of volcanic activity were first compiled. We include some volcanoes on our list of 'potentially active' volcanoes that do not exactly fit these criteria because geologic evidence suggests that they have been active within the last few thousand years and as such, although not historically active, they retain a potential for hazardous activity that requires careful monitoring. As geologic understanding of Alaska's volcanoes improves through additional fieldwork and modern radiometric-dating techniques, our list of "active" volcanoes will undoubtedly evolve. A case in point from 2006: Fourpeaked Mountain, thought not to have erupted in the Holocene, produced a phreatic eruption in the fall of 2006. It now ranks as an historically active volcano, despite not appearing on the list prior to 2006. 
Table 4a. Compilation by year of volcanoes included in an Alaska Volcano Observatory Annual Summary, 1992-2011.

[Volcanoes are presented in geographical order from northeast to southwest along the Wrangell-Aleutian volcanic arc and north to south along Kamchatka and the Kurile Islands. Prior to 1995 and after 2010, Alaska Volcano Observatory did not report on Russian volcanoes]

\begin{tabular}{|c|c|}
\hline \multicolumn{2}{|c|}{ Volcanoes mentioned } \\
\hline Alaskan & Russian \\
\hline \multicolumn{2}{|c|}{1992} \\
\hline \multicolumn{2}{|l|}{$\begin{array}{l}\text { Spurr/Crater Peak } \\
\text { Iliamna } \\
\text { Redoubt } \\
\text { Mageik (Katmai Group) } \\
\text { Westdahl } \\
\text { Akutan } \\
\text { Bogoslof } \\
\text { Seguam }\end{array}$} \\
\hline \multicolumn{2}{|c|}{1993} \\
\hline \multicolumn{2}{|l|}{$\begin{array}{l}\text { Churchill } \\
\text { Sanford } \\
\text { Spurr/Crater Peak } \\
\text { Veniaminof } \\
\text { Shishaldin } \\
\text { Makushin } \\
\text { Seguam } \\
\text { Kliuchef (Atka) } \\
\text { Kanaga }\end{array}$} \\
\hline \multicolumn{2}{|c|}{1994} \\
\hline $\begin{array}{l}\text { Sanford } \\
\text { Iliamna } \\
\text { Katmai Group (Martin, } \\
\quad \text { Mageik, Trident) } \\
\text { Veniaminof } \\
\text { Kupreanof } \\
\text { Shishaldin } \\
\text { Makushin } \\
\text { Cleveland } \\
\text { Kanaga }\end{array}$ & \\
\hline \multicolumn{2}{|c|}{1995} \\
\hline $\begin{array}{l}\text { Katmai Group (Martin) } \\
\text { Veniaminof } \\
\text { Shishaldin } \\
\text { Makushin } \\
\text { Kliuchef (Atka) } \\
\text { Kanaga }\end{array}$ & $\begin{array}{l}\text { Bezymianny } \\
\text { Karymsky }\end{array}$ \\
\hline \multicolumn{2}{|c|}{1996} \\
\hline $\begin{array}{l}\text { Wrangell } \\
\text { Iliamna } \\
\text { Katmai Group (Martin, } \\
\quad \text { Mageik, Trident, Mount } \\
\quad \text { Katmai) } \\
\text { Pavlof } \\
\text { Shishaldin } \\
\text { Westdahl } \\
\text { Akutan } \\
\text { Amukta } \\
\text { Korovin (Atka) } \\
\text { Kanaga }\end{array}$ & $\begin{array}{l}\text { Klyuchevskoy } \\
\text { Bezymianny } \\
\text { Karymsky } \\
\text { Avachinsky } \\
\text { Mutnovsky } \\
\text { Alaid (Kurile Islands) }\end{array}$ \\
\hline
\end{tabular}

\begin{tabular}{|c|c|}
\hline \multicolumn{2}{|c|}{ Volcanoes mentioned } \\
\hline Alaskan & Russian \\
\hline \multicolumn{2}{|c|}{1997} \\
\hline $\begin{array}{l}\text { Wrangell } \\
\text { Sanford } \\
\text { Shrub Mud } \\
\text { Iliamna } \\
\text { Katmai Group (Martin, } \\
\quad \text { Mageik, Snowy, Kukak) } \\
\text { Chiginagak } \\
\text { Pavlof } \\
\text { Shishaldin } \\
\text { Okmok } \\
\text { Cleveland } \\
\text { Amukta }\end{array}$ & $\begin{array}{l}\text { Sheveluch } \\
\text { Klyuchevskoy } \\
\text { Bezymianny } \\
\text { Karymsky } \\
\text { Alaid (Kurile Islands) }\end{array}$ \\
\hline \multicolumn{2}{|c|}{1998} \\
\hline $\begin{array}{l}\text { Shrub Mud } \\
\text { Augustine } \\
\text { Becharof Lake } \\
\text { Chiginagak } \\
\text { Shishaldin } \\
\text { Akutan } \\
\text { Korovin (Atka) }\end{array}$ & $\begin{array}{l}\text { Sheveluch } \\
\text { Klyuchevskoy } \\
\text { Bezymianny } \\
\text { Karymsky }\end{array}$ \\
\hline \multicolumn{2}{|c|}{1999} \\
\hline $\begin{array}{l}\text { Wrangell } \\
\text { Shrub Mud } \\
\text { Iliamna } \\
\text { Veniaminof } \\
\text { Pavlof } \\
\text { Shishaldin } \\
\text { Vsevidof }\end{array}$ & $\begin{array}{l}\text { Sheveluch } \\
\text { Klyuchevskoy } \\
\text { Bezymianny } \\
\text { Karymsky }\end{array}$ \\
\hline \multicolumn{2}{|c|}{2000} \\
\hline $\begin{array}{l}\text { Wrangell } \\
\text { Katmai Group (Snowy) } \\
\text { Chiginagak } \\
\text { Shishaldin }\end{array}$ & $\begin{array}{l}\text { Sheveluch } \\
\text { Klyuchevskoy } \\
\text { Bezymianny } \\
\text { Karymsky } \\
\text { Mutnovsky }\end{array}$ \\
\hline \multicolumn{2}{|c|}{2001} \\
\hline $\begin{array}{l}\text { Katmai Group } \\
\quad \text { (Snowy/Kukak) } \\
\text { Pavlof } \\
\text { Frosty } \\
\text { Shishaldin } \\
\text { Makushin } \\
\text { Okmok } \\
\text { Cleveland } \\
\text { Great Sitkin }\end{array}$ & $\begin{array}{l}\text { Sheveluch } \\
\text { Klyuchevskoy } \\
\text { Bezymianny } \\
\text { Karymsky } \\
\text { Avachinsky }\end{array}$ \\
\hline
\end{tabular}


Table 4a. Compilation by year of volcanoes included in an Alaska Volcano Observatory Annual Summary, 1992-2011.—Continued

[Volcanoes are presented in geographical order from northeast to southwest along the Wrangell-Aleutian volcanic arc and north to south along Kamchatka and the Kurile Islands. Prior to 1995, Alaska Volcano Observatory did not report on Russian volcanoes]

\begin{tabular}{|c|c|}
\hline \multicolumn{2}{|c|}{ Volcanoes mentioned } \\
\hline Alaskan & Russian \\
\hline \multicolumn{2}{|c|}{2002} \\
\hline $\begin{array}{l}\text { Wrangell } \\
\text { Katmai Group (Martin, } \\
\text { Mageik) } \\
\text { Veniaminof } \\
\text { Mt. Hague (Emmons } \\
\text { Lake Caldera) } \\
\text { Shishaldin } \\
\text { Great Sitkin }\end{array}$ & $\begin{array}{l}\text { Sheveluch } \\
\text { Klyuchevskoy } \\
\text { Bezymianny } \\
\text { Karymsky }\end{array}$ \\
\hline \multicolumn{2}{|c|}{2003} \\
\hline $\begin{array}{l}\text { Wrangell } \\
\text { Redoubt } \\
\text { Iliamna } \\
\text { Augustine } \\
\text { Katmai Group (Mageik) } \\
\text { Veniaminof } \\
\text { Pavlof } \\
\text { Mt. Hague (Emmons } \\
\text { Lake Caldera) } \\
\text { Shishaldin } \\
\text { Akutan }\end{array}$ & $\begin{array}{l}\text { Sheveluch } \\
\text { Klyuchevskoy } \\
\text { Bezymianny } \\
\text { Karymsky } \\
\text { Alaid } \\
\text { Chikurachki }\end{array}$ \\
\hline \multicolumn{2}{|c|}{2004} \\
\hline $\begin{array}{l}\text { Mt. Crillon (non-volcanic } \\
\text { peak) } \\
\text { Mount Spurr } \\
\text { Katmai Group (Martin) } \\
\text { Veniaminof } \\
\text { Shishaldin } \\
\text { Westdahl }\end{array}$ & $\begin{array}{l}\text { Sheveluch } \\
\text { Klyuchevskoy } \\
\text { Bezymianny } \\
\text { Karymsky } \\
\text { Chirinkotan (Kuriles) }\end{array}$ \\
\hline \multicolumn{2}{|c|}{2005} \\
\hline $\begin{array}{l}\text { Mount Spurr } \\
\text { Iliamna } \\
\text { Augustine } \\
\text { Katmai Group (Martin, } \\
\quad \text { Mageik, Trident) } \\
\text { Chiginagak } \\
\text { Aniakchak } \\
\text { Veniaminof } \\
\text { Pavlof/Mt. Hague } \\
\text { Shishaldin } \\
\text { Cleveland } \\
\text { Korovin } \\
\text { Kasatochi } \\
\text { Tanaga }\end{array}$ & $\begin{array}{l}\text { Sheveluch } \\
\text { Klyuchevskoy } \\
\text { Bezymianny } \\
\text { Karymsky } \\
\text { Avachinsky } \\
\text { Mutnovsky } \\
\text { Ebeko } \\
\text { Chikurachki }\end{array}$ \\
\hline
\end{tabular}

\begin{tabular}{|c|c|}
\hline \multicolumn{2}{|c|}{ Volcanoes mentioned } \\
\hline Alaskan & Russian \\
\hline \multicolumn{2}{|c|}{2006} \\
\hline $\begin{array}{l}\text { Klawasi } \\
\text { Mount Spurr } \\
\text { Augustine } \\
\text { Fourpeaked } \\
\text { Katmai Group (Martin, } \\
\quad \text { Mageik, Trident) } \\
\text { Veniaminof } \\
\text { Cleveland } \\
\text { Korovin } \\
\text { Kasatochi }\end{array}$ & $\begin{array}{l}\text { Sheveluch } \\
\text { Klyuchevskoy } \\
\text { Bezymianny } \\
\text { Karymsky } \\
\text { Ebeko } \\
\text { Severgin } \\
\text { Berga }\end{array}$ \\
\hline \multicolumn{2}{|c|}{2007} \\
\hline $\begin{array}{l}\text { Redoubt } \\
\text { Augustine } \\
\text { Fourpeaked } \\
\text { Veniaminof } \\
\text { Pavlof } \\
\text { Akutan } \\
\text { Cleveland } \\
\text { Korovin }\end{array}$ & $\begin{array}{l}\text { Sheveluch } \\
\text { Klyuchevskoy } \\
\text { Bezymianny } \\
\text { Karymsky } \\
\text { Gorely and Mutnovsky } \\
\text { Chikurachki } \\
\text { Berga }\end{array}$ \\
\hline \multicolumn{2}{|c|}{2008} \\
\hline $\begin{array}{l}\text { Redoubt } \\
\text { Aniakchak } \\
\text { Veniaminof } \\
\text { Shishaldin } \\
\text { Okmok } \\
\text { Cleveland } \\
\text { Kasatochi }\end{array}$ & $\begin{array}{l}\text { Sheveluch } \\
\text { Klyuchevskoy } \\
\text { Bezymianny } \\
\text { Karymsky } \\
\text { Koryaksky } \\
\text { Gorely and Mutnovsky } \\
\text { Chikurachki } \\
\text { Tyatya }\end{array}$ \\
\hline \multicolumn{2}{|c|}{2009} \\
\hline $\begin{array}{l}\text { Sanford } \\
\text { Redoubt } \\
\text { Fourpeaked } \\
\text { Aniakchak } \\
\text { Veniaminof } \\
\text { Shishaldin } \\
\text { Okmok } \\
\text { Cleveland }\end{array}$ & $\begin{array}{l}\text { Sheveluch } \\
\text { Kluchevskoy } \\
\text { Bezymianny } \\
\text { Kizimen } \\
\text { Karymsky } \\
\text { Koryaksky } \\
\text { Gorley } \\
\text { Ebeko } \\
\text { Sarychev } \\
\text { Raikoke }\end{array}$ \\
\hline
\end{tabular}


Table 4a. Compilation by year of volcanoes included in an Alaska Volcano Observatory Annual Summary, 1992-2011.—Continued

[Volcanoes are presented in geographical order from northeast to southwest along the Wrangell-Aleutian volcanic arc and north to south along Kamchatka and the Kurile Islands. Prior to 1995, Alaska Volcano Observatory did not report on Russian volcanoes]

\begin{tabular}{lc}
\hline \multicolumn{1}{c}{ Alaskan } & \multicolumn{1}{c}{ Russian } \\
\hline & 2010 \\
\hline Wrangell & Sheveluch \\
Mt. Sanford & Klyuchevskoy \\
Redoubt & Bezymianny \\
Fourpeaked & Kizimen \\
Katmai Group & Karymsky \\
Becharof Lake & Gorely \\
Aniakchak & Ekarma \\
Veniaminof & \\
Westdahl & \\
Makushin & \\
Cleveland & \\
Kasatochi & \\
\hline
\end{tabular}

Aniakchak

Okmok

Cleveland 
Table 4b. Compilation by volcano for particular years included in an Alaska Volcano Observatory Annual Summary, 1992-2011.

[Suspect Volcanic Activity (SVA) is defined as a report of eruption or possible eruption that is normal fumarolic activity or non-volcanic phenomena, such as weather related. PIREP, pilot weather report]

\begin{tabular}{|c|c|c|}
\hline Volcano & $\begin{array}{c}\text { Year } \\
\text { mentioned }\end{array}$ & Type of activity \\
\hline \multicolumn{3}{|r|}{ Alaska (east to west) } \\
\hline Churchill & 1993 & SVA, anomalous seismicity \\
\hline Wrangell & $\begin{array}{l}1996 \\
1997 \\
1999 \\
2000 \\
2002 \\
2003 \\
2007 \\
2010\end{array}$ & $\begin{array}{l}\text { SVA, steam plume } \\
\text { SVA, steam plume } \\
\text { SVA, steaming and phreatic ash emission } \\
\text { SVA, steam plumes } \\
\text { SVA, suspicious clouds, redistributed ash } \\
\text { SVA, anomalous clouds } \\
\text { Triggered seismicity, vapor clouds, wind-blown ash } \\
\text { Anomalous clouds }\end{array}$ \\
\hline Sanford & $\begin{array}{l}1993 \\
1994 \\
1997 \\
2009 \\
2010\end{array}$ & $\begin{array}{l}\text { SVA, reported steam plume likely from avalanche } \\
\text { SVA, reported steam plume likely from avalanche } \\
\text { SVA, large steam cloud from SW face } \\
\text { Anomalous cloud } \\
\text { Anomalous cloud from SW face }\end{array}$ \\
\hline Shrub Mud & $\begin{array}{l}1997 \\
1998 \\
1999\end{array}$ & $\begin{array}{l}\text { Eruption; energetic ejection of saline mud and } \mathrm{CO}_{2} \\
\text { Eruption continues; ejection of saline mud and } \mathrm{CO}_{2} \\
\text { Eruption continues; ejection of saline mud and } \mathrm{CO}_{2}\end{array}$ \\
\hline Klawasi Mud & 2006 & Possible new mud vent \\
\hline Spurr & $\begin{array}{l}1992 \\
1993 \\
2004 \\
2005 \\
2006\end{array}$ & $\begin{array}{l}\text { Subplinian eruptions; ash, pyroclastic flows, lahars } \\
\text { SVA, glacial outburst produces seismicity } \\
\text { Heat flux to summit; lahars; cauldron develops } \\
\text { Continued heat to summit; cauldron evolves } \\
\text { Continued heat to summit; cauldron evolves }\end{array}$ \\
\hline Redoubt & $\begin{array}{l}1992 \\
2003 \\
2007 \\
2008 \\
2009 \\
2010\end{array}$ & $\begin{array}{l}\text { SVA, steam plume from still-cooling dome } \\
\text { SVA, anomalous weather cloud } \\
\text { Possible steaming and increased thermal flux } \\
\text { Pre-eruption increase in gas emissions and thermal flux from summit crater } \\
\text { Major magmatic eruption } \\
\text { Vapor and gas clouds; brief uptick in seismicity }\end{array}$ \\
\hline Iliamna & $\begin{array}{l}1992 \\
1994 \\
1996 \\
1997 \\
1999 \\
2003 \\
2005\end{array}$ & $\begin{array}{l}\text { SVA, PIREP of large steam plume, media frenzy } \\
\text { SVA, vigorous steam plume, avalanche } \\
\text { Intense seismicity related to magmatic intrusion } \\
\text { SVA; anomalous seismic swarm; avalanche } \\
\text { SVA, avalanche } \\
\text { SVA, avalanche } \\
\text { SVA, rock avalanche }\end{array}$ \\
\hline Augustine & $\begin{array}{l}1998 \\
2005 \\
2006 \\
2007\end{array}$ & $\begin{array}{l}1986 \text { dome spine partially collapses, generates mudflow } \\
\text { Precursory activity prior to eruption in early } 2006 \\
\text { Explosive and effusive eruption } \\
\text { Strong seismicity and steam plumes }\end{array}$ \\
\hline Fourpeaked & $\begin{array}{l}2006 \\
2007 \\
2009 \\
2010\end{array}$ & $\begin{array}{l}\text { Phreatic eruption } \\
\text { Ongoing fumarolic emissions, seismicity } \\
\text { Continued decrease in steam and gas emissions } \\
\text { Decreasing fumarolic emissions, sporadic earthquake swarms }\end{array}$ \\
\hline
\end{tabular}


Table 4b. Compilation by volcano for particular years included in an Alaska Volcano Observatory Annual Summary, 1992-2011.-Continued

[Suspect Volcanic Activity (SVA) is defined as a report of eruption or possible eruption that is normal fumarolic activity or non-volcanic phenomena, such as weather related. PIREP, pilot weather report]

\begin{tabular}{|c|c|c|}
\hline Volcano & $\begin{array}{c}\text { Year } \\
\text { mentioned }\end{array}$ & Type of activity \\
\hline \multicolumn{3}{|c|}{ Alaska (east to west)—Continued } \\
\hline \multicolumn{3}{|l|}{ Katmai Group } \\
\hline Mageik & 1992 & SVA, anomalous cloud \\
\hline Martin/Mageik/Trident & 1994 & SVA, plume-like cloud \\
\hline Martin & 1995 & SVA, large steam plume \\
\hline Martin/Mageik/Trident/Mount Katmai & 1996 & SVA, anomalous seismicity \\
\hline Martin/Mageik/Snowy/Kukak & 1997 & SVA, PIREPS of ash and steam plumes \\
\hline Snowy & 2000 & SVA, steaming hole in glacier \\
\hline Snowy/Kukak & 2001 & SVA, steaming hole in glacier \\
\hline Martin/Mageik & 2002 & SVA, steam plume \\
\hline Mageik & 2003 & SVA, steaming, large cloud of re-suspended ash \\
\hline \multirow[t]{3}{*}{ Martin } & 2004 & SVA, large steam plume \\
\hline & 2006 & Earthquake swarm \\
\hline & 2010 & Re-suspended ash \\
\hline Martin/Mageik/Trident & 2005 & SVA, steam cloud, re-suspended ash, new crater? \\
\hline \multirow[b]{2}{*}{ Becharof Lake } & 1998 & SVA, intense seismic swarm and inflationary episode \\
\hline & 2010 & Earthquake swarm \\
\hline \multirow[t]{4}{*}{ Chiginagak } & 1997 & Minor eruptive activity, new fumarole field \\
\hline & 1998 & SVA, continuation of increased fumarolic activity \\
\hline & 2000 & SVA, steam emissions from fumarole field \\
\hline & 2005 & Heat to summit; acidic flood; cauldron develops \\
\hline \multirow[t]{5}{*}{ Aniakchak } & 2005 & SVA, anomalous seismicity, thermal anomaly \\
\hline & 2008 & Weather related noise on seismic stations \\
\hline & 2009 & Anomalous seismicity; network outage \\
\hline & 2010 & Low frequency earthquake swarms \\
\hline & 2011 & Anomalous seismicity \\
\hline \multirow[t]{13}{*}{ Veniaminof } & 1993 & Low-level eruption and lava flows \\
\hline & 1994 & Strombolian eruption and lava flows \\
\hline & 1995 & Strombolian eruptions \\
\hline & 1999 & SVA, extreme discharge and turbid river \\
\hline & 2002 & Low-level phreatic eruptions \\
\hline & 2003 & Low-level phreatic eruptions \\
\hline & 2004 & Weak phreatic and Strombolian eruption \\
\hline & 2005 & Intermittent phreatic and Strombolian eruption \\
\hline & 2006 & Intermittent phreatic and Strombolian eruption \\
\hline & 2007 & Decline in vapor plumes \\
\hline & 2008 & Weak phreatic emissions and vapor plumes \\
\hline & 2009 & Minor phreatic eruptions \\
\hline & 2010 & Sporadic seismicity, vapor plumes \\
\hline Kupreanof & 1994 & SVA, PIREP of unusual steam plume \\
\hline \multirow[t]{6}{*}{ Pavlof } & 1996 & Strombolian eruption \\
\hline & 1997 & Strombolian eruption concludes \\
\hline & 1999 & SVA, summit snow melt, ash dustings, steam plumes \\
\hline & 2001 & SVA, steaming, possible ash, sulfur smell \\
\hline & 2005 & SVA, mis-located steam plume \\
\hline & 2007 & Strombolian eruption, lava flows, lahars \\
\hline \multirow[t]{3}{*}{ Hague (Emmons Lake Caldera) } & 2002 & SVA, increase in fumarolic activity in summit crater \\
\hline & 2003 & SVA, crater lake drains, refills, drains \\
\hline & 2005 & SVA, steam plume \\
\hline Frosty & 2001 & SVA, rock fall avalanches \\
\hline
\end{tabular}


Table 4b. Compilation by volcano for particular years included in an Alaska Volcano Observatory Annual Summary, 1992-2011.-Continued

[Suspect Volcanic Activity (SVA) is defined as a report of eruption or possible eruption that is normal fumarolic activity or non-volcanic phenomena, such as weather related. PIREP, pilot weather report]

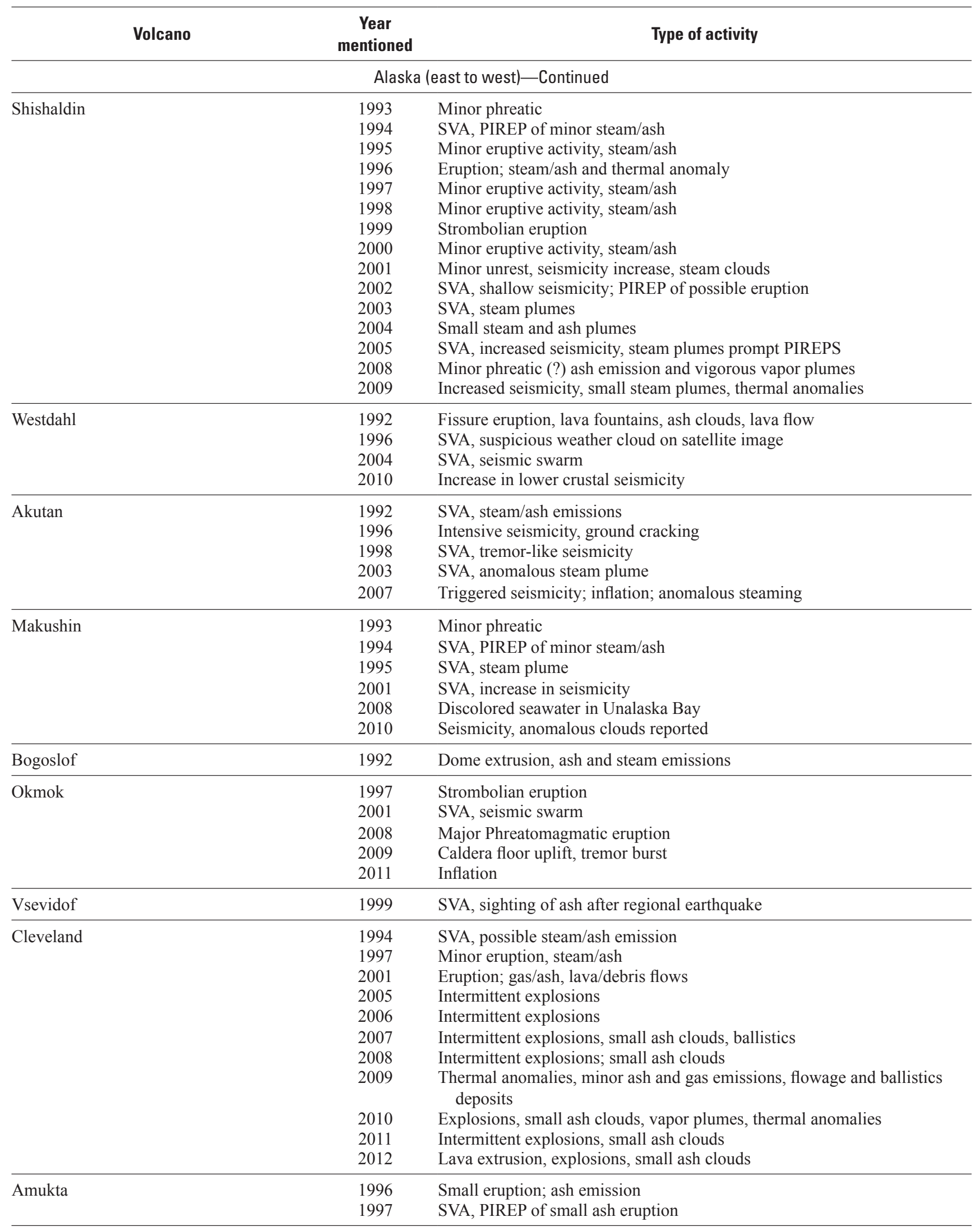


Table 4b. Compilation by volcano for particular years included in an Alaska Volcano Observatory Annual Summary, 1992-2011.-Continued

[Suspect Volcanic Activity (SVA) is defined as a report of eruption or possible eruption that is normal fumarolic activity or non-volcanic phenomena, such as weather related. PIREP, pilot weather report]

\begin{tabular}{|c|c|c|}
\hline Volcano & $\begin{array}{c}\text { Year } \\
\text { mentioned }\end{array}$ & Type of activity \\
\hline Seguam/Pyre Peak & $\begin{array}{l}1992 \\
1993\end{array}$ & $\begin{array}{l}\text { Minor eruptive activity, steam/ash emissions } \\
\text { Fissure eruption produces lava flow and ash cloud }\end{array}$ \\
\hline Kliuchef (Atka) & $\begin{array}{l}1993 \\
1995\end{array}$ & $\begin{array}{l}\text { SVA, audible rumbling, strong sulfur odor } \\
\text { SVA, large steam plume, strong sulfur odor }\end{array}$ \\
\hline Korovin (Atka) & $\begin{array}{l}1996 \\
1998 \\
2005 \\
2006 \\
2007\end{array}$ & $\begin{array}{l}\text { SVA, PIREP of ash cloud, suspicious cloud on satellite image } \\
\text { Eruption; explosions and ash fall } \\
\text { Minor eruption, steam and ash } \\
\text { Seismic swarms, uplift, increased fumarolic activity } \\
\text { Seismic swarms; fumarolic activity }\end{array}$ \\
\hline Kasatochi & $\begin{array}{l}2005 \\
2006 \\
2008 \\
2010\end{array}$ & $\begin{array}{l}\text { SVA, unusual bubbling; floating scum on crater lake } \\
\text { Continued bubbling in intracaldera lake } \\
\text { Major explosive eruption } \\
\text { Fumarolic emission, diffuse degassing, coastal erosion }\end{array}$ \\
\hline Great Sitkin & $\begin{array}{l}2001 \\
2002\end{array}$ & $\begin{array}{l}\text { SVA, anomalous seismicity } \\
\text { SVA, seismic swarm, tremor }\end{array}$ \\
\hline Kanaga & $\begin{array}{l}1993 \\
1994 \\
1995 \\
1996\end{array}$ & $\begin{array}{l}\text { SVA, increased steaming } \\
\text { Eruption; steam/ash and lava flow } \\
\text { Minor eruptive activity, steam/ash and lava } \\
\text { Possible eruption and ash emission }\end{array}$ \\
\hline Tanaga & 2005 & SVA, anomalous seismicity, including a period of tremor \\
\hline \multicolumn{3}{|c|}{ Kamchatka and northern Kurile Islands (north to south) — activity through 2010} \\
\hline Sheveluch & $\begin{array}{l}1997 \\
1998 \\
1999 \\
2000 \\
2001 \\
2002 \\
2003 \\
2004 \\
2005 \\
2006 \\
2007 \\
2008 \\
2009 \\
2010\end{array}$ & $\begin{array}{l}\text { Dome extrusion } \\
\text { Lava dome growth } \\
\text { Lava dome growth and collapse, ash } \\
\text { Lava dome growth, ash } \\
\text { Lava dome growth and collapse, ash } \\
\text { Lava dome growth, ash, pyroclastic flows } \\
\text { Lava dome growth, ash, pyroclastic flows, lahar } \\
\text { Lava dome growth, pyroclastic flows, lahars, ash } \\
\text { Lava dome growth, dome collapse, pyroclastic flows, ash } \\
\text { Lava dome growth, dome collapse, explosions } \\
\text { Lava dome growth, dome collapse, explosions } \\
\text { Lava dome growth, dome collapse, explosions } \\
\text { Lava dome growth, dome collapse, explosions } \\
\text { Lava dome growth, dome collapse, explosions }\end{array}$ \\
\hline Klyuchevskoy & $\begin{array}{l}1996 \\
1997 \\
1998 \\
1999 \\
2000 \\
2001 \\
2002 \\
2003 \\
2004 \\
2005 \\
2006 \\
2007 \\
2008 \\
2009 \\
2010\end{array}$ & $\begin{array}{l}\text { Gas/ash eruption } \\
\text { Gas/ash eruption } \\
\text { Gas/ash eruption } \\
\text { Gas/ash eruption } \\
\text { Vulcanian explosions } \\
\text { Fumarolic plume } \\
\text { Elevated seismicity, gas-rich explosion } \\
\text { Elevated seismicity, ash explosion, Strombolian activity } \\
\text { Elevated seismicity } \\
\text { Strombolian eruption, lava flows, lahars } \\
\text { Increased seismicity, thermal anomaly, no eruption } \\
\text { Ash emission, Strombolian lava fountaining, lava flows } \\
\text { Strombolian lava fountaining, lava flows, lahars, phreatic explosions } \\
\text { Strombolian, Vulcanian activity, lava flow production, ash falls } \\
\text { Strombolian lava fountaining, explosions, lava flows, lahars }\end{array}$ \\
\hline
\end{tabular}


Table 4b. Compilation by volcano for particular years included in an Alaska Volcano Observatory Annual Summary, 1992-2011.-Continued

[Suspect Volcanic Activity (SVA) is defined as a report of eruption or possible eruption that is normal fumarolic activity or non-volcanic phenomena, such as weather related. PIREP, pilot weather report]

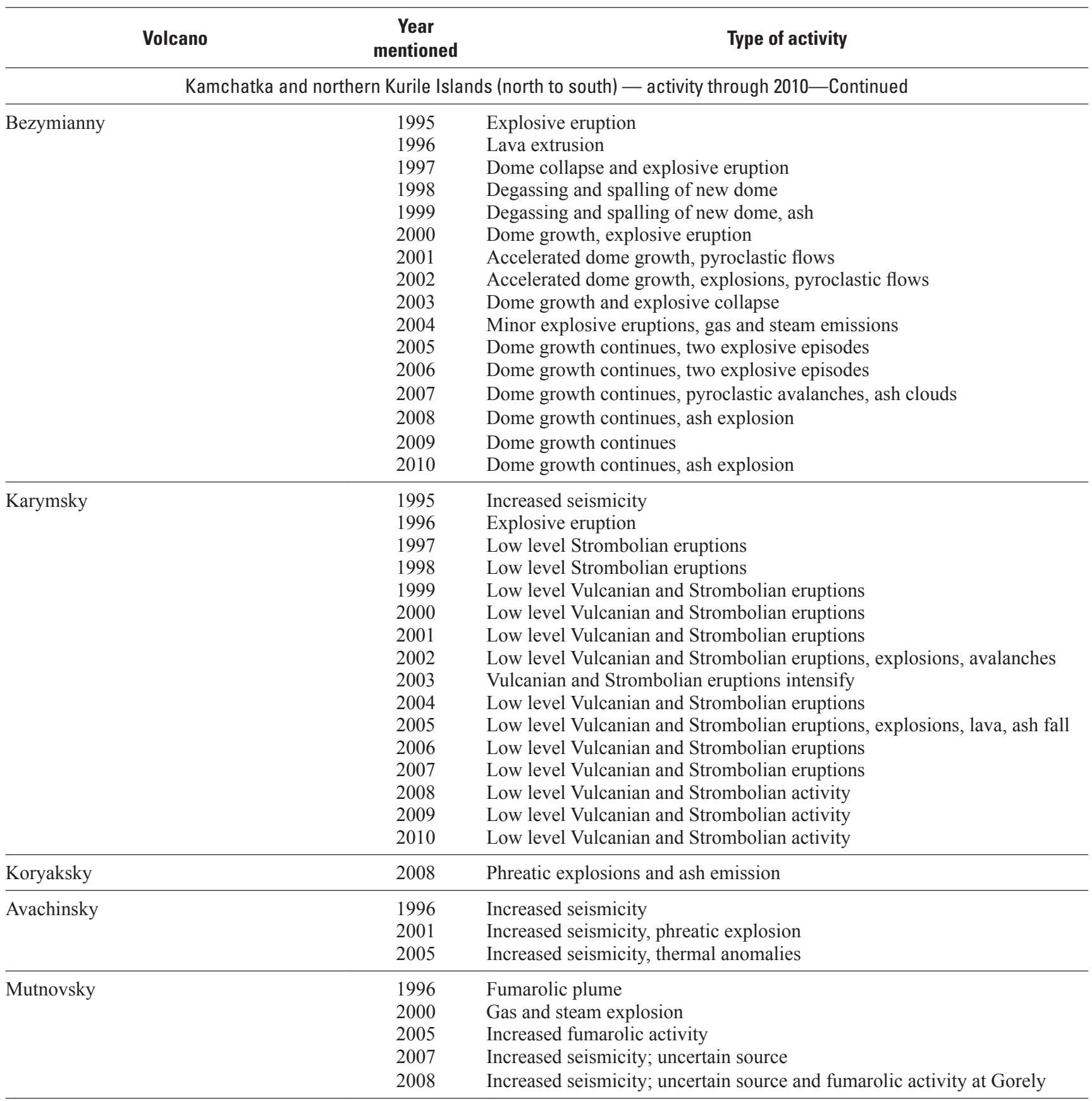


Table 4b. Compilation by volcano for particular years included in an Alaska Volcano Observatory Annual Summary, 1992-2011.-Continued

[Suspect Volcanic Activity (SVA) is defined as a report of eruption or possible eruption that is normal fumarolic activity or non-volcanic phenomena, such as weather related. PIREP, pilot weather report]

\begin{tabular}{|c|c|c|}
\hline Volcano & $\begin{array}{c}\text { Year } \\
\text { mentioned }\end{array}$ & Type of activity \\
\hline \multicolumn{3}{|c|}{ Kamchatka and northern Kurile Islands (north to south) — activity through 2010 —Continued } \\
\hline Gorley & $\begin{array}{l}2007 \\
2008 \\
2009 \\
2010\end{array}$ & $\begin{array}{l}\text { Increased seismicity; uncertain source } \\
\text { Increased seismicity; uncertain source and fumarolic activity at Gorely } \\
\text { Increased seismicity, thermal output, degassing }\end{array}$ \\
\hline Alaid (Kurile Islands) & $\begin{array}{l}1996 \\
1997\end{array}$ & $\begin{array}{l}\text { Ash plume } \\
\text { SVA }\end{array}$ \\
\hline Ebeko & $\begin{array}{l}2005 \\
2006\end{array}$ & $\begin{array}{l}\text { Increased fumarolic activity and phreatic eruptions } \\
\text { Increased fumarolic activity }\end{array}$ \\
\hline Chikurachki & $\begin{array}{l}2003 \\
2005 \\
2007 \\
2008\end{array}$ & $\begin{array}{l}\text { Stombolian and Vulcanian eruption, ash fall } \\
\text { Brief explosion produces ash and ash fall } \\
\text { Ash explosions } \\
\text { Explosions and limited ash clouds }\end{array}$ \\
\hline Severgin & 2006 & Phreatic or fumarolic activity \\
\hline Sarychev & 2009 & Major magmatic eruption; check location in table \\
\hline Chirinkotan & 2004 & Brief, low-level steam, gas, and ash emission \\
\hline Ekarma & 2010 & Phreatic eruption, lahar detected after the fact; sheck location in table \\
\hline Berga & $\begin{array}{l}2006 \\
2007\end{array}$ & $\begin{array}{l}\text { Phreatic or fumarolic activity } \\
\text { Possible phreatic or fumarolic activity }\end{array}$ \\
\hline Tyatya & 2008 & Possible increase in fumarolic activity \\
\hline
\end{tabular}


Table 4c. Citations for Alaska Volcano Observatory Annual Summary reports, 1992-2011.

\begin{tabular}{|c|c|}
\hline Year & Citation \\
\hline 1992 & $\begin{array}{l}\text { McGimsey, R.G., Neal, C.A., and Doukas, M.P., 1995, Volcanic activity in Alaska: } \\
\text { Summary of events and response of the Alaska Volcano Observatory 1992: U.S. } \\
\text { Geological Survey Open-File Report 95-83, } 26 \text { p. }\end{array}$ \\
\hline 1993 & $\begin{array}{l}\text { Neal, C.A., McGimsey, R.G., and Doukas, M.P., 1996, } 1993 \text { Volcanic activity } \\
\text { in Alaska: Summary of events and response of the Alaska Volcano Observatory: } \\
\text { U.S. Geological Survey Open-File Report 96-24, } 21 \text { p. }\end{array}$ \\
\hline 1994 & $\begin{array}{l}\text { Neal, C.A., Doukas, M.P., and McGimsey, R.G., 1995, } 1994 \text { Volcanic activity in } \\
\text { Alaska: Summary of events and response of the Alaska Volcano Observatory: } \\
\text { U.S. Geological Survey Open-File Report 95-271, } 20 \text { p. }\end{array}$ \\
\hline 1995 & $\begin{array}{l}\text { McGimsey, R.G., and Neal, C.A., 1996, } 1995 \text { Volcanic activity in Alaska and } \\
\text { Kamchatka: Summary of events and response of the Alaska Volcano Observatory: } \\
\text { U.S. Geological Survey Open-File Report 96-738, } 22 \text { p. }\end{array}$ \\
\hline
\end{tabular}

1996 Neal, C.A., and McGimsey, R.G., 1997, 1996 Volcanic activity in Alaska and Kamchatka: Summary of events and response of the Alaska Volcano Observatory: U.S. Geological Survey Open-File Report 97-433, 34 p.

1997 McGimsey, R.G., and Wallace, K.L., 1999, 1997 Volcanic activity in Alaska and Kamchatka: Summary of events and response of the Alaska Volcano Observatory: U.S. Geological Survey Open-File Report 99-448, 42 p.

1998 McGimsey, R.G., Neal, C.A., and Girina, Olga, 2003, 1998 Volcanic activity in Alaska and Kamchatka: Summary of events and response of the Alaska Volcano Observatory: U.S. Geological Survey Open-File Report 03-423, 35 p.

1999 McGimsey, R. G., Neal, C. A., and Girina, Olga, 2004a, 1999 Volcanic activity in Alaska and Kamchatka: Summary of events and response of the Alaska Volcano Observatory: U.S. Geological Survey Open-File Report OF 2004-1033, 49 p.

2000 Neal, C.A., McGimsey, R.G., and Chubarova, Olga, 2004, 2000 Volcanic activity in Alaska and Kamchatka: Summary of events and response of the Alaska Volcano Observatory: U.S. Geological Survey Open-File Report 2004-1034, 37 p.

2001 McGimsey, R.G., Neal, C.A., and Girina, Olga, 2004b, 2001 Volcanic activity in Alaska and Kamchatka: Summary of events and response of the Alaska Volcano Observatory: U.S. Geological Survey Open-File Report 2004-1453, 57 p.

2002 Neal, C.A., McGimsey, R.G., and Girina, Olga, 2005, 2002 Volcanic activity in Alaska and Kamchatka: Summary of events and response of the Alaska Volcano Observatory: U.S. Geological Survey Open-File Report 2004-1058, 55 p.

2003 McGimsey, R.G., Neal, C.A., and Girina, Olga, 2005, 2003 Volcanic activity in Alaska and Kamchatka: Summary of events and response of the Alaska Volcano Observatory: U.S. Geological Survey Open-File Report 2005-1310, 62 p.

2004 Neal, C.A., McGimsey, R.G., Dixon, J.P., and Melnikov, Dmitry, 2005, 2004 Volcanic activity in Alaska and Kamchatka: Summary of events and response of the Alaska Volcano Observatory: U.S. Geological Survey Open-File Report 2005-1308, $71 \mathrm{p}$.

2005 McGimsey, R.G., Neal, C.A., Dixon, J.P., Ushakov, Sergey, 2007, 2005 Volcanic activity in Alaska, Kamchatka, and the Kurile Islands: Summary of events and response of the Alaska Volcano Observatory: U.S. Geological Survey Scientific Investigations Report 2007-5269, 94 p.

2006 Neal, C.A., McGimsey, R.G., Dixon, J.P., Manevich, Alexander, and Rybin, Alexander, 2009, 2006 Volcanic activity in Alaska, Kamchatka, and the Kurile Islands: Summary of events and response of the Alaska Volcano Observatory: U.S. Geological Survey Scientific Investigations Report 2008-5214, 102 p. http://pubs.er.usgs.gov/publication/ofr9583

http://pubs.usgs.gov/of/1996/0024/

http://pubs.usgs.gov/of/1995/0271/

http://pubs.usgs.gov/of/1996/0738/

http://pubs.usgs.gov/of/1997/0433/

http://pubs.usgs.gov/of/1999/0448/

http://pubs.usgs.gov/of/2003/of03-423/

http://pubs.usgs.gov/of/2004/1033/

http://pubs.usgs.gov/of/2004/1034/

http://pubs.usgs.gov/of/2004/1453/

http://pubs.usgs.gov/of/2004/1058/

http://pubs.usgs.gov/of/2005/1310/

http://pubs.usgs.gov/of/2005/1308/

http://pubs.usgs.gov/sir/2007/5269/

http://pubs.usgs.gov/sir/2008/5214/ 
Table 4c. Citations for Alaska Volcano Observatory Annual Summary reports, 1992-2011.-Continued

\begin{tabular}{|c|c|c|}
\hline Year & Citation & URL \\
\hline 2007 & $\begin{array}{l}\text { McGimsey, R.G., Neal, C.A., Dixon, J.P., Malik, Nataliya, and Chibisova, Marina, } \\
\text { 2011, } 2007 \text { Volcanic activity in Alaska, Kamchatka, and the Kurile Islands: } \\
\text { Summary of events and response of the Alaska Volcano Observatory: U.S. } \\
\text { Geological Survey Scientific Investigations Report 2010-5242, } 110 \text { p. }\end{array}$ & http://pubs.usgs.gov/sir/2010/5242/ \\
\hline 2008 & $\begin{array}{l}\text { Neal, C.A., McGimsey, R.G., Dixon, J.P., Cameron, C.E., Nuzhdaev, A.E., and } \\
\text { Chibisova, M., 2011, } 2008 \text { Volcanic activity in Alaska, Kamchatka, and the Kurile } \\
\text { Islands: Summary of events and response of the Alaska Volcano Observatory: } \\
\text { U.S. Geological Survey Scientific Investigations Report 2010-5243, } 87 \text { p. }\end{array}$ & http://pubs.usgs.gov/sir/2010/5243/ \\
\hline 2009 & $\begin{array}{l}\text { McGimsey, R.G., Neal, C.A., Girina, O.A., Chibisova, Marina, and Rybin, } \\
\text { Alexander, 2013, } 2009 \text { Volcanic activity in Alaska, Kamchatka, and the Kurile } \\
\text { Islands: Summary of events and response of the Alaska Volcano Observatory: } \\
\text { U.S. Geological Survey Scientific Investigations Report 2013-5213, } 125 \text { p. }\end{array}$ & http://pubs.usgs.gov/sir/2013/5213/ \\
\hline 2010 & $\begin{array}{l}\text { Neal, C.A., Herrick, J., Girina, O., Chibisova, M., Rybin, A., McGimsey, R., and } \\
\text { Dixon, J., 2013, } 2010 \text { Volcanic activity in Alaska, Kamchatka, and the Kurile } \\
\text { Islands: Summary of events and response of the Alaska Volcano Observatory: } \\
\text { U.S. Geological Survey Scientific Investigations Report 2013-5034, } 76 \text { p. }\end{array}$ & http://pubs.usgs.gov/sir/2013/5034/ \\
\hline 2011 & $\begin{array}{l}\text { McGimsey, R.G., Maharrey, J.Z., and Neal, C.A., 2014, } 2011 \text { Volcanic activity in } \\
\text { Alaska-Summary of events and response of the Alaska Volcano Observatory: } \\
\text { U.S. Geological Survey Scientific Investigations Report 2014-5159, } 50 \text { p. }\end{array}$ & http://pubs.usgs.gov/sir/2014/5159 \\
\hline
\end{tabular}




\section{Volcanic Activity in Alaska, Northeast to Southwest Along Aleutian Arc}

\section{Aniakchak Volcano}

CAVW\# 1102-09-

$56^{\circ} 54^{\prime} \mathrm{N} 158^{\circ} 13^{\prime} \mathrm{W}$

$1,341 \mathrm{~m}(4,400 \mathrm{ft})$

Alaska Peninsula

DRAINAGE OF MAAR LAKE, destructive downstream flooding

In late August 2011, AVO was alerted by Troy Hamon, Chief of Resource Management, Katmai National Park, that the larger of two maar crater lakes on the caldera floor of Aniakchak Volcano had recently drained, leaving a gaping notch in the crater rim. The flood had ravaged the downstream drainage and alluvial fan as well as the upper reaches of the Aniakchak River, which drains Surprise Lake and the eastern caldera floor through The Gates. During September 8-9, 2011, authors Tina Neal and Game McGimsey, accompanied by Troy Hamon (on September 8), visited the site to document and study the activity.

Aniakchak caldera formed about 3,600 years ago in a colossal eruption (Neal and others, 2001). Subsequent eruptive activity has occurred from many intracaldera vents, including eruptions that formed two maar craters, subsequently filling with water, that are situated on the southeastern caldera floor between the central Vent Mountain cone and the sheer inner east caldera wall (fig. 2). The larger maar is about $450 \mathrm{~m}$ $(1,476 \mathrm{ft})$ by $515 \mathrm{~m}(1,690 \mathrm{ft})$, as measured rim to rim in Google EarthTM imagery (fig. 3); the smaller maar is $170 \mathrm{~m}$
$(558 \mathrm{ft})$ in diameter at the rim. The maars formed subsequent to the draining of a caldera-wide lake, at least 1,000 years before present (BP), but they may be much younger, perhaps less than 400 years old.

Geological investigations in 1992 documented that a low point on the maar rim was about 10-15 $\mathrm{m}(33-49 \mathrm{ft})$ above the lake surface, although higher strand lines were visible in the deposits surrounding the maar, one of which was located nearly at the low point (figs. 3 and 4; Neal and others, 2001). On the outboard flank of the maar-rim low-point, a seeppiping through the maar crater wall deposits to the lakeoccurred near the base of the flank and formed the headwater for the stream that courses between a prominent lava flow from Vent Mountain and the colluvial slopes extending out from the eastern caldera wall (fig. 3). This stream flows about $1.7 \mathrm{~km}(1 \mathrm{mi})$ northward from its source at the maar to the Aniakchak River immediately inboard of The Gates, a prominent notch in the caldera wall through which the river drains the entire caldera (fig. 2). 


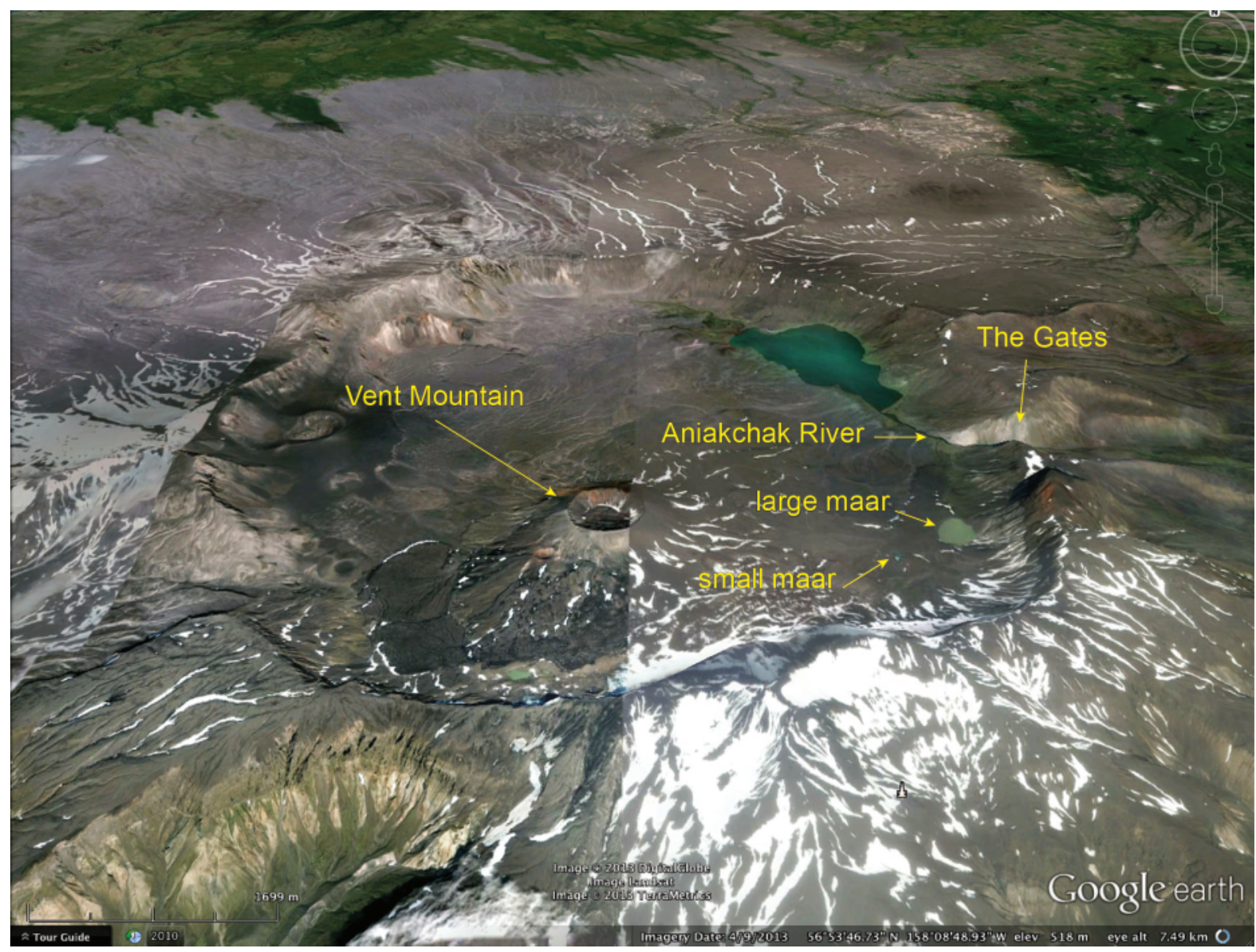

Figure 2. Oblique Google EarthTM view of Aniakchak caldera looking to the north. The larger of two maar craters lie on the southeastern floor of the caldera adjacent to the steep eastern caldera wall. The caldera is about $10 \mathrm{~km}(6.2$ $\mathrm{mi})$ in diameter. The larger maar is $450 \mathrm{~m}(1,476 \mathrm{ft})$ by $515 \mathrm{~m}(1,690 \mathrm{ft})$, and the smaller maar is $170 \mathrm{~m}(558 \mathrm{ft})$ in diameter at the rim. The maar craters lie along the caldera ring fracture system. This Google EarthTM image was taken on August 8, 2007, prior to the maar flood. AVO database image at URL: http://www.avo.alaska.edu/images/image. php?id $=57531$. 


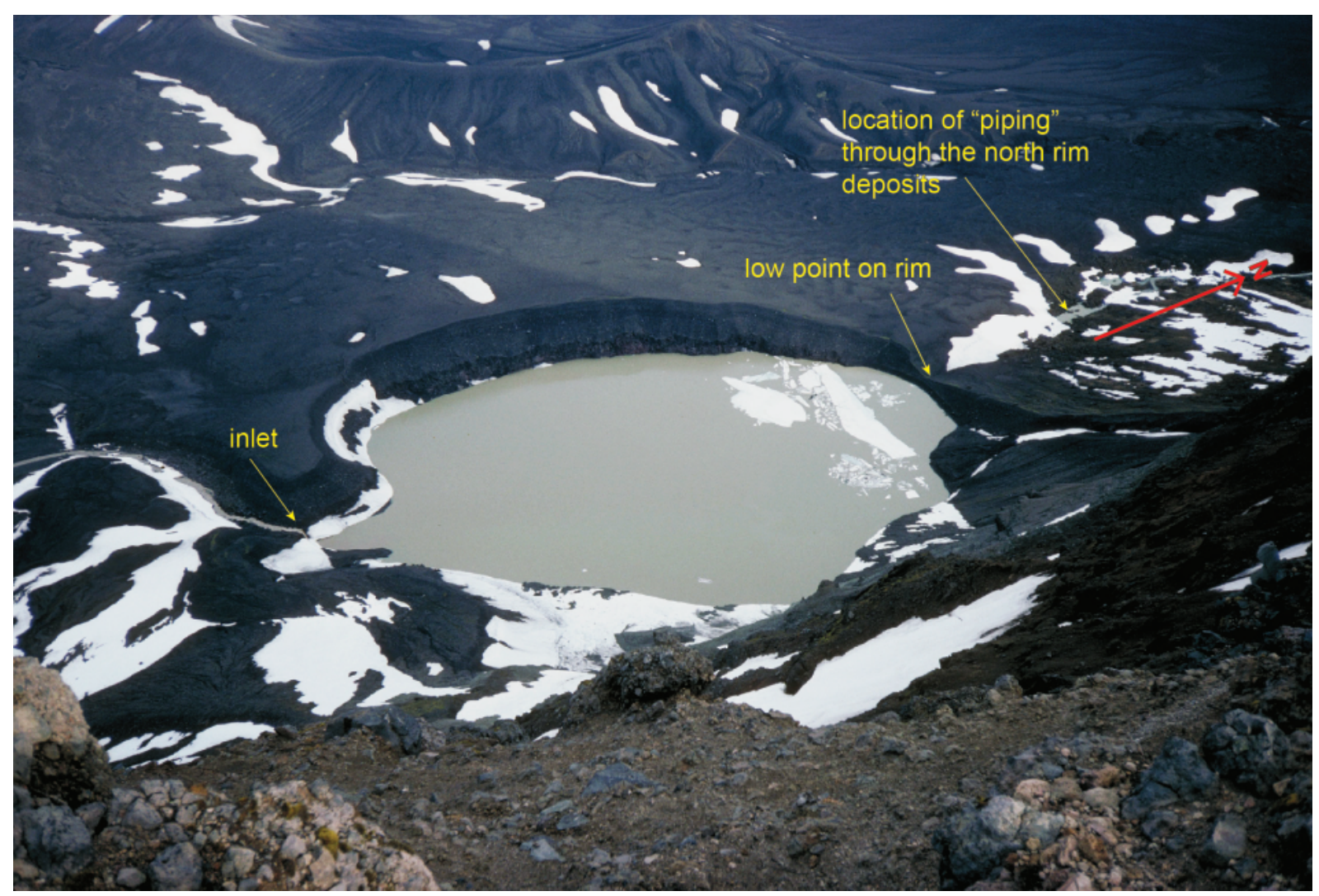

Figure 3. Photograph showing larger of two maar craters on the southeastern floor of Aniakchak caldera as viewed from the southeastern caldera rim. The crater is about $450 \mathrm{~m}(1,476 \mathrm{ft})$ by $515 \mathrm{~m}(1,690 \mathrm{ft})$, measured rim to rim. The low point on the rim is where flood water exited the crater, flowing northward $1.7 \mathrm{~km}(1 \mathrm{mi})$ to the Aniakchak River. The southeastern corner of the caldera drains into the lake and lake level is maintained by "piping" through the northern rim deposits. Photograph by C.A. Neal, AVO/USGS, June 29, 1992. AVO database image at URL: http:// www.avo.alaska.edu/images/image.php?id=10405.

The maar breakout flood scoured and excavated the channel and banks of the stream north of the maar, cutting deeply into the colluvial slopes on the eastern side of the drainage (fig. 4). The previously well-vegetated alluvial fan near The Gates was entirely covered by flood deposits up to $1.5 \mathrm{~m}$ (4.9 ft) thick (fig. 5). Clumps of vegetation, up to about $1 \mathrm{~m}(3.3 \mathrm{ft})$ in length, were strewn on and within the deposit. The Aniakchak River was pressed even more tightly against the northern wall opposite the fan, causing some bank erosion, and the downstream river channel, sand bars, and banks were noticeably disrupted for more than $20 \mathrm{~km}$ (12.4 mi) beyond The Gates (fig. 6). The area of the breach on the maar rim was dramatically altered as downcutting progressed until a new base level was established. Discharge through the outflow at the northern end of the maar now approximates inflow at the southern end (primarily at the waterfall, fig. 3).
Field measurements and observations indicate that the maar lake level declined $4.65 \mathrm{~m}(15.3 \mathrm{ft})$ from its recent high stand (determined from photographs taken July 18, 2010 (fig. 7), as well as measurements made in the field in September 2011); this high stand was below the low-point on the northern rim where the breach occurred. The volume of water lost was at least $645,000 \mathrm{~m}^{3}\left(844,000 \mathrm{yds}^{3}\right)$, but this represents a minimum volume because there is no way of knowing how much additional water flooded into the maar immediately prior to initiation of the breakout flooding.

Based on analysis of photographs, weather data, and observations from a local guide, the maar flood likely occurred between late July and late September 2010. Evidence of recent flooding on the southern floor and wall of the caldera, which drain into the maar, suggests that the maar flood was likely caused by the rapid and voluminous 


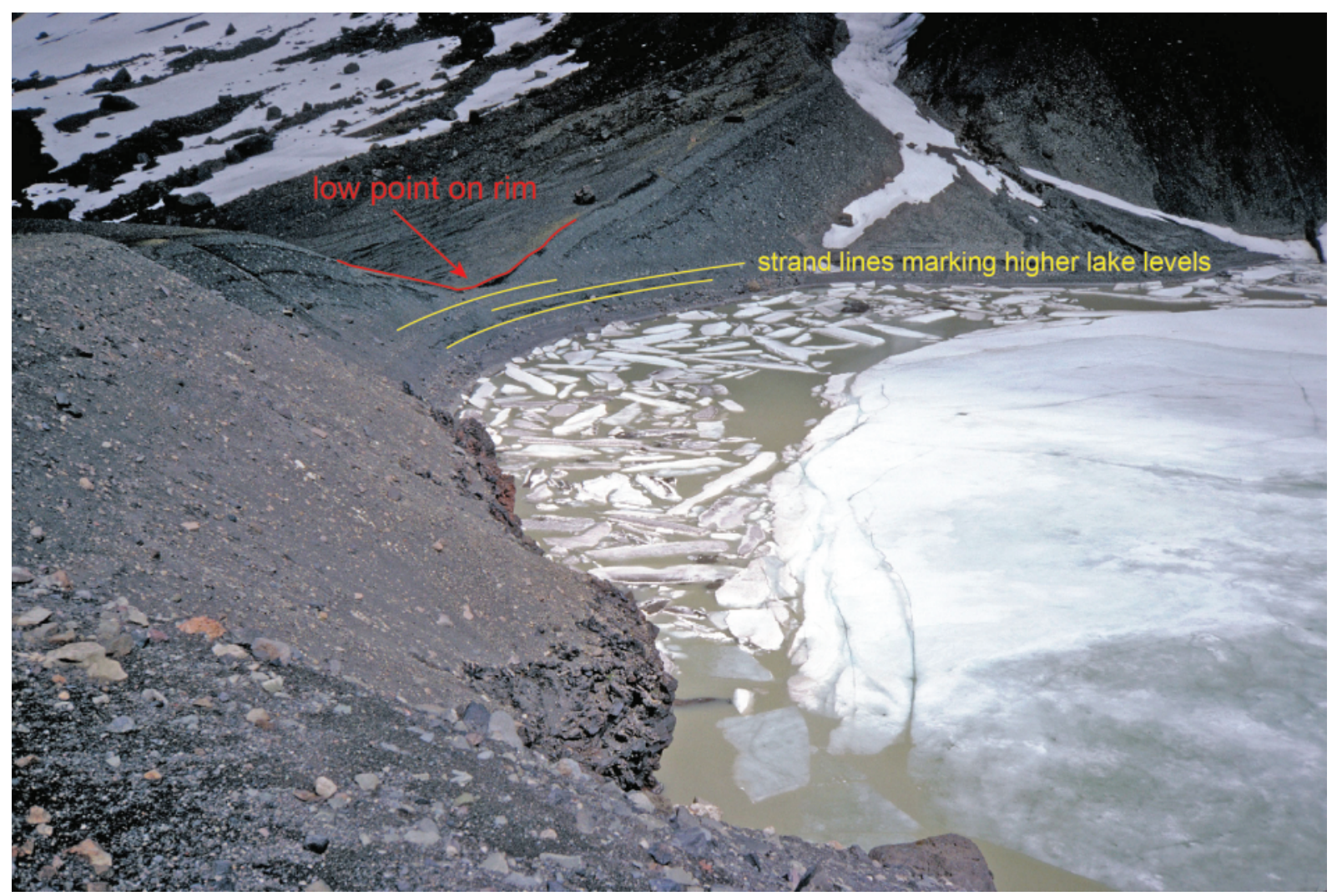

Figure 4. Photograph of the northwestern rim of the maar; view is toward the low point on the northern rim. Several strand lines mark higher stands of the lake, and the low point is likely a former spillway. Photograph by C.A. Neal, AVO/ USGS, June 24, 1992. AVO database image at URL: http://www.avo.alaska.edu/images/image. php?id=10402.

influx of water into the maar lake during a period of unusually heavy rainfall. The lake level rose rapidly and the low point on the maar rim may have been reached and spilled over. The increased hydraulic head may have simultaneously initiated failure of the crater wall at that location where groundwater seepage and possibly piping within the mantling deposit was occurring. The heavy precipitation may have further increased saturation of the deposit. Once the breach began, by either rapid downcutting, or structural failure of the wall, or both, rapid release of water occurred, resulting in a short-lived but massive flood down the drainage to - and then down - the Aniakchak River (figs. 8 and 9 ).
Aniakchak is a circular caldera $10 \mathrm{~km}(6.2 \mathrm{mi})$ in diameter and as deep as $1 \mathrm{~km}(3,280 \mathrm{ft})$ from the rim to the caldera floor. The caldera formed during a huge eruption about 3,600 years ago (Miller and Smith, 1987; Dreher and others, 2005). Numerous lava domes, lava flows, and scoria cones occupy the interior of the caldera (Neal and others, 2001); the largest intracaldera cone is Vent Mountain, $2.5 \mathrm{~km}(1.5 \mathrm{mi})$ in diameter and rising $430 \mathrm{~m}(1,410 \mathrm{ft})$ above the floor of the caldera. The only historical eruption of Aniakchak, a powerfully explosive event that covered a large portion of the eastern Alaska Peninsula with ash, occurred in 1931 (Hubbard, 1931; Neal and others, 2001; Nicholson, 2003). 


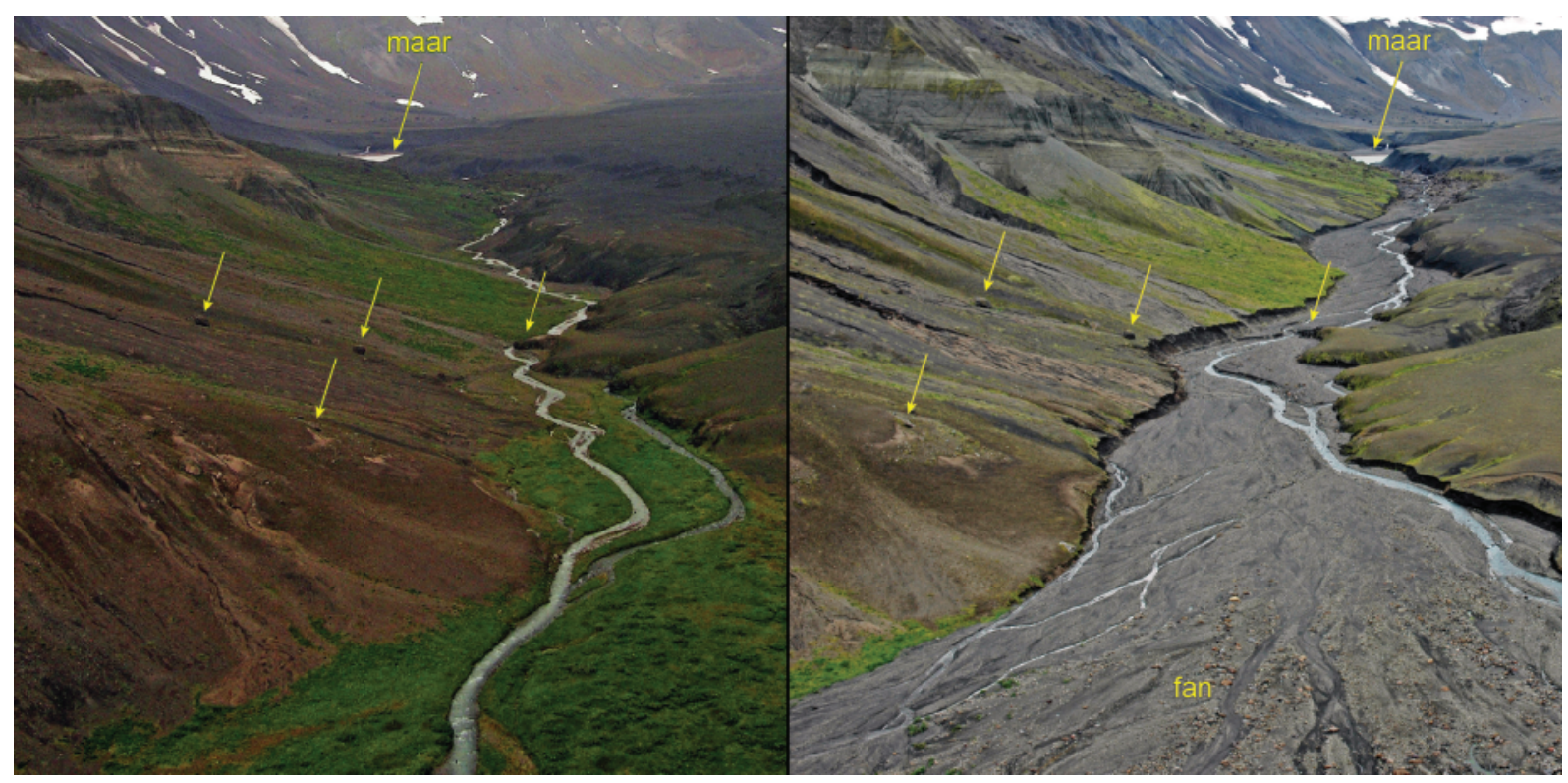

Figure 5. Photographs of drainage between the maar and the Aniakchak River before and after the flood event showing displacement of the stream channel and erosion of the flood plain and fan. Arrows point to several prominent features common on both images. Image on the left was taken by Ryan Rumelhart, NPS, August 4, 2005. AVO database image at URL: http://www.avo. alaska.edu/images/image.php?id=57541. Image on the right was taken by Game McGimsey, AVO/USGS, September 7, 2011. AVO database image at URL: http://www.avo.alaska.edu/images/image.php?id=57551.

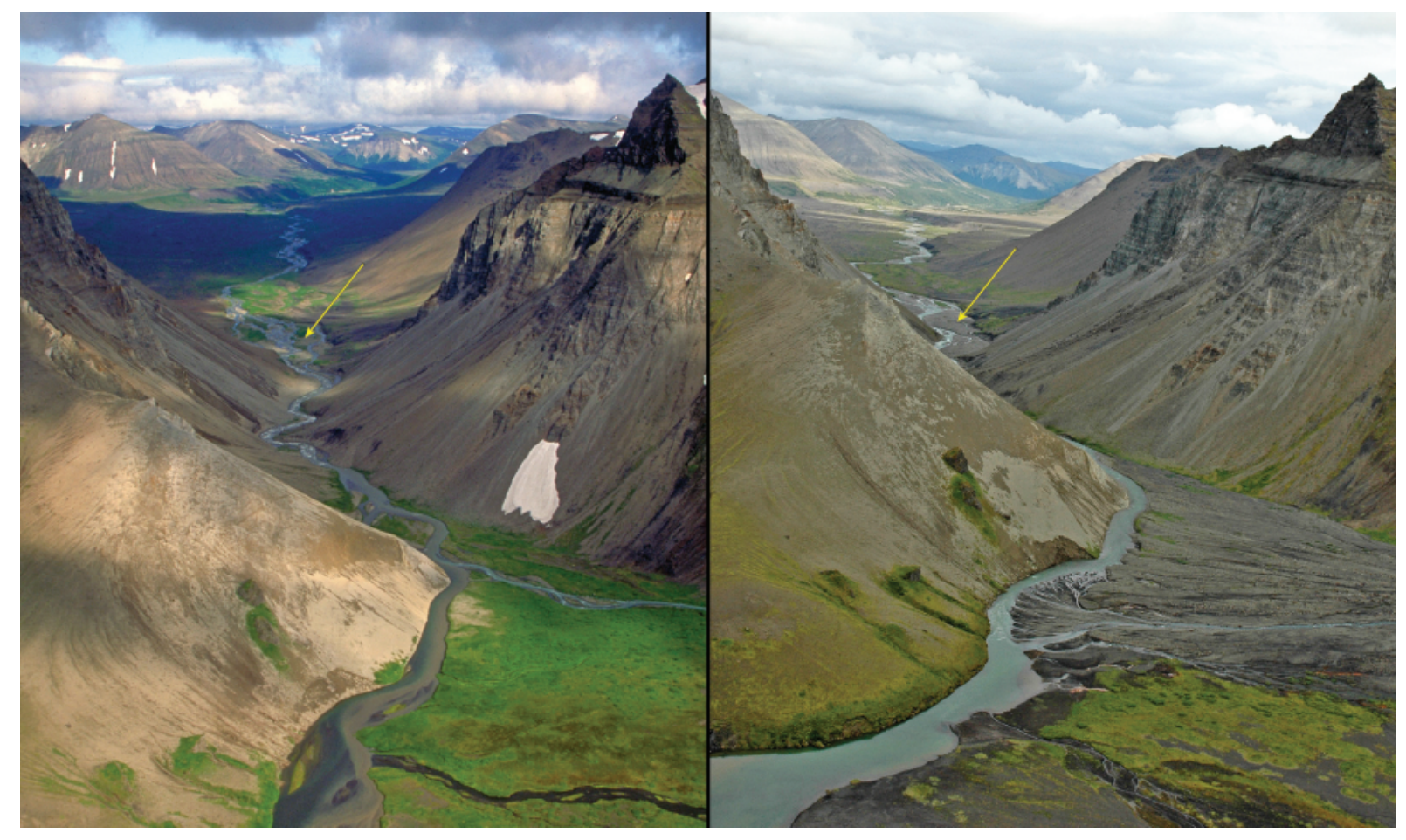

Figure 6. Comparative aerial oblique views east showing the Aniakchak River exiting the caldera through The Gates. Image on the left was taken prior to the maar breakout flood; note the lush green alluvial fan center right, where streams draining the eastern side of the caldera join the Aniakchak River. Image on the right shows the effects of the 2010 flood on the alluvial fan as well as to the Aniakchak River (arrow), where the flood plain was swept of vegetation and flood debris was deposited; the alluvial fan has been inundated by the maar flood debris, and the course of the main stream has shifted to the western margin of the fan (down in the image). Image on the left was taken by Game McGimsey, AVO/USGS, July 22, 1993. AVO database image at URL: http://www.avo.alaska.edu/images/image.php?id=10534. Image on the right was taken by Game McGimsey, AVO/USGS, September 7, 2011. AVO database image at URL: http://www.avo.alaska.edu/images/image.php?id=57561. 


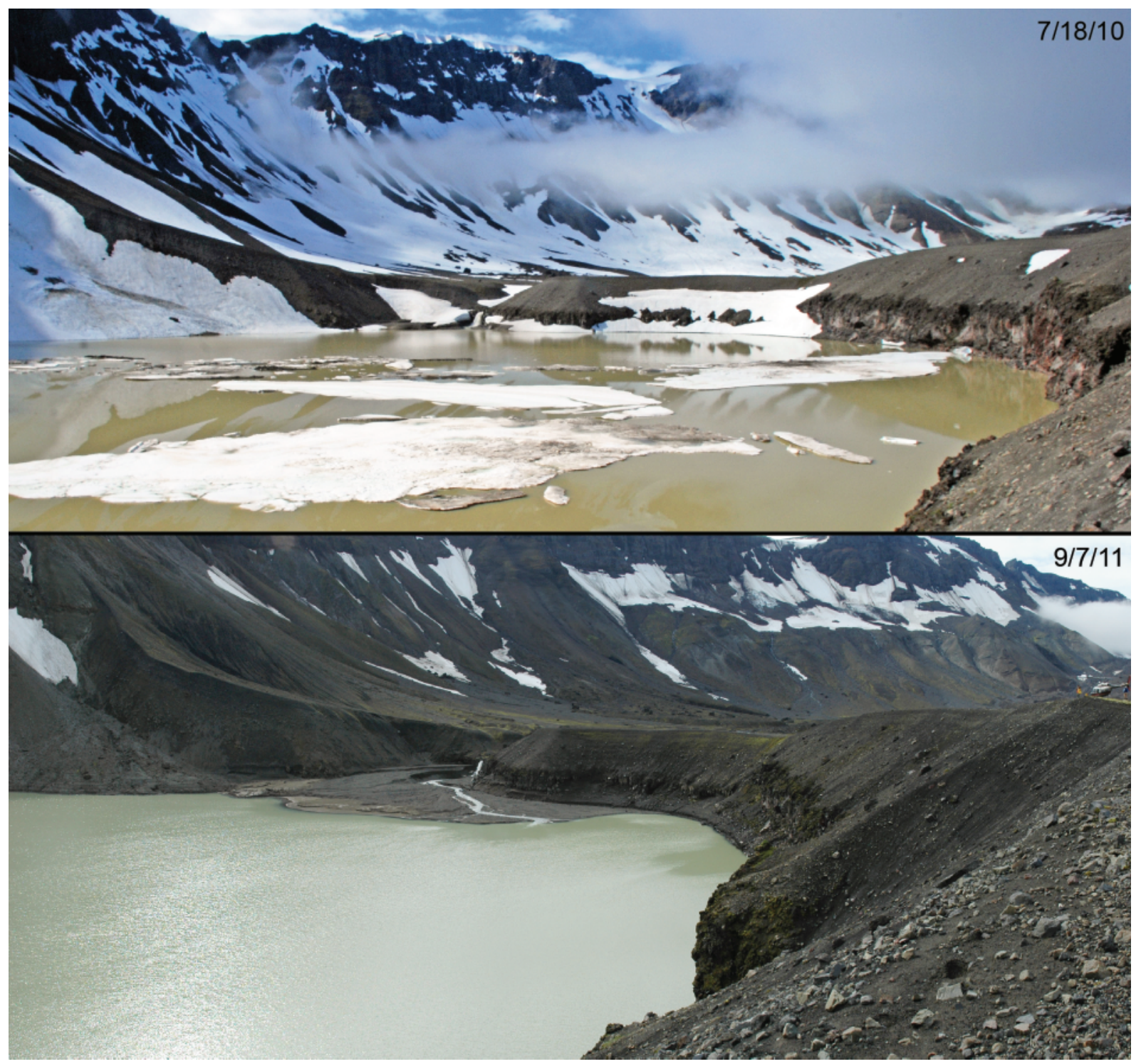

Figure 7. Comparative images of the large maar in Aniakchak caldera. Lake level dropped declined at least $4.65 \mathrm{~m}$ $(15.3 \mathrm{ft})$ when the northern rim of the maar failed, releasing at least $645,000 \mathrm{~m}^{3}\left(844,000 \mathrm{yds}^{3}\right)$ of lake water. Image on top was taken by Tom O'Keefe, July 18, 2010. AVO database image at URL: http://www.avo.alaska.edu/images/image. php?id=57571. Image on bottom was taken by Game McGimsey, AVO/USGS, September 7, 2011. AVO database image at URL: http://www.avo.alaska.edu/images/image.php?id=57581. 


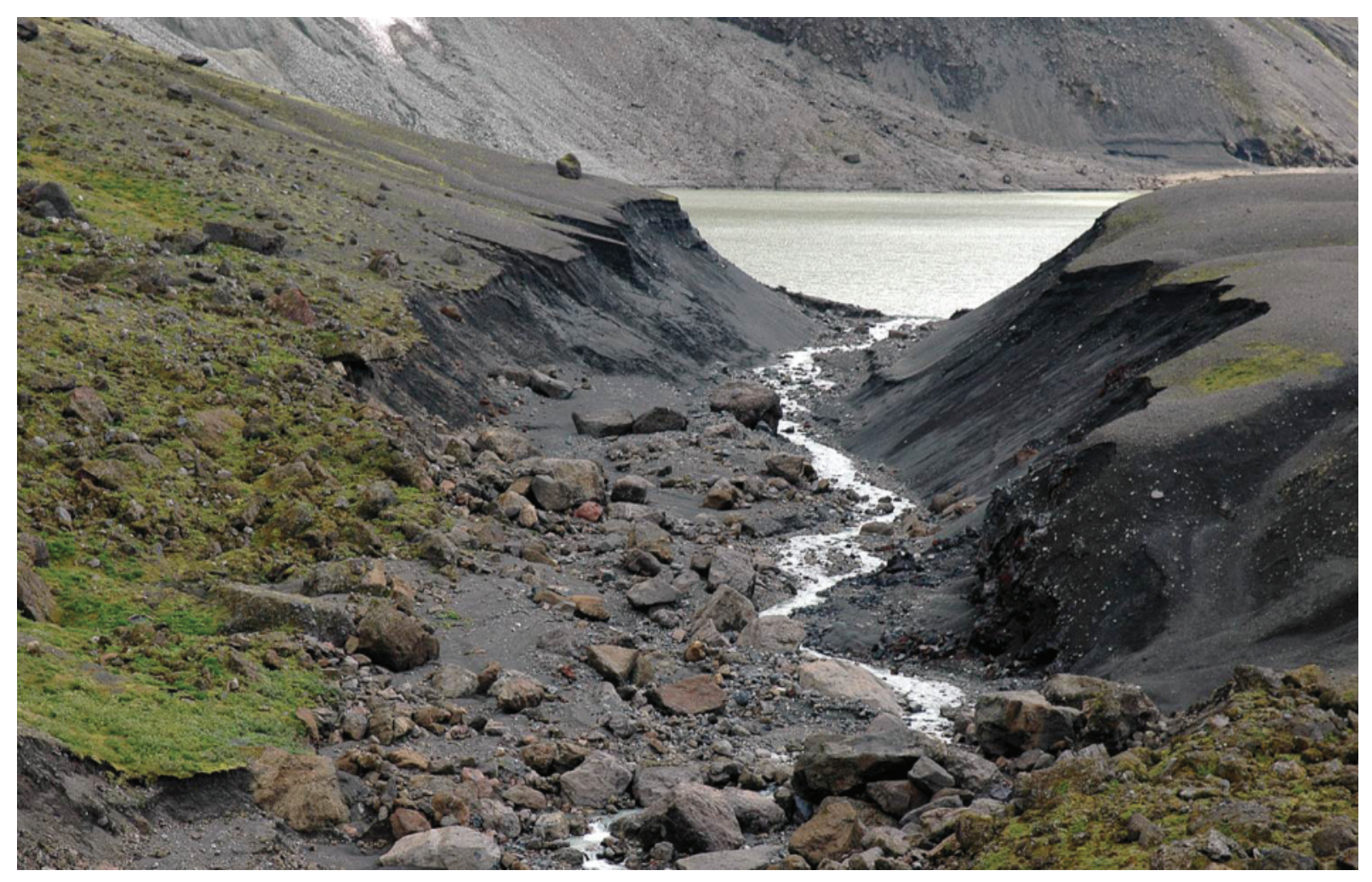

Figure 8. View south of the breach in the northern rim of the maar crater lake on the eastern floor of Aniakchak caldera. The coarse debris in the middle ground is the toe of a rockslide that had mantled the base of the colluvial eastern slope of the eastern caldera wall. The flood winnowed the fine material and left the coarser boulders as lag. The breach occurred at the lowest point on the maar rim immediately to the right of the large boulder above the left side of cut. Groundwater seepage or piping through the rim deposits had been occurring at the area shown at the extreme bottom of image. Photograph by Game McGimsey, AVO/USGS, September 7, 2011. AVO database image at URL: http:// www.avo.alaska.edu/images/image. $p h p ? i d=57591$. 


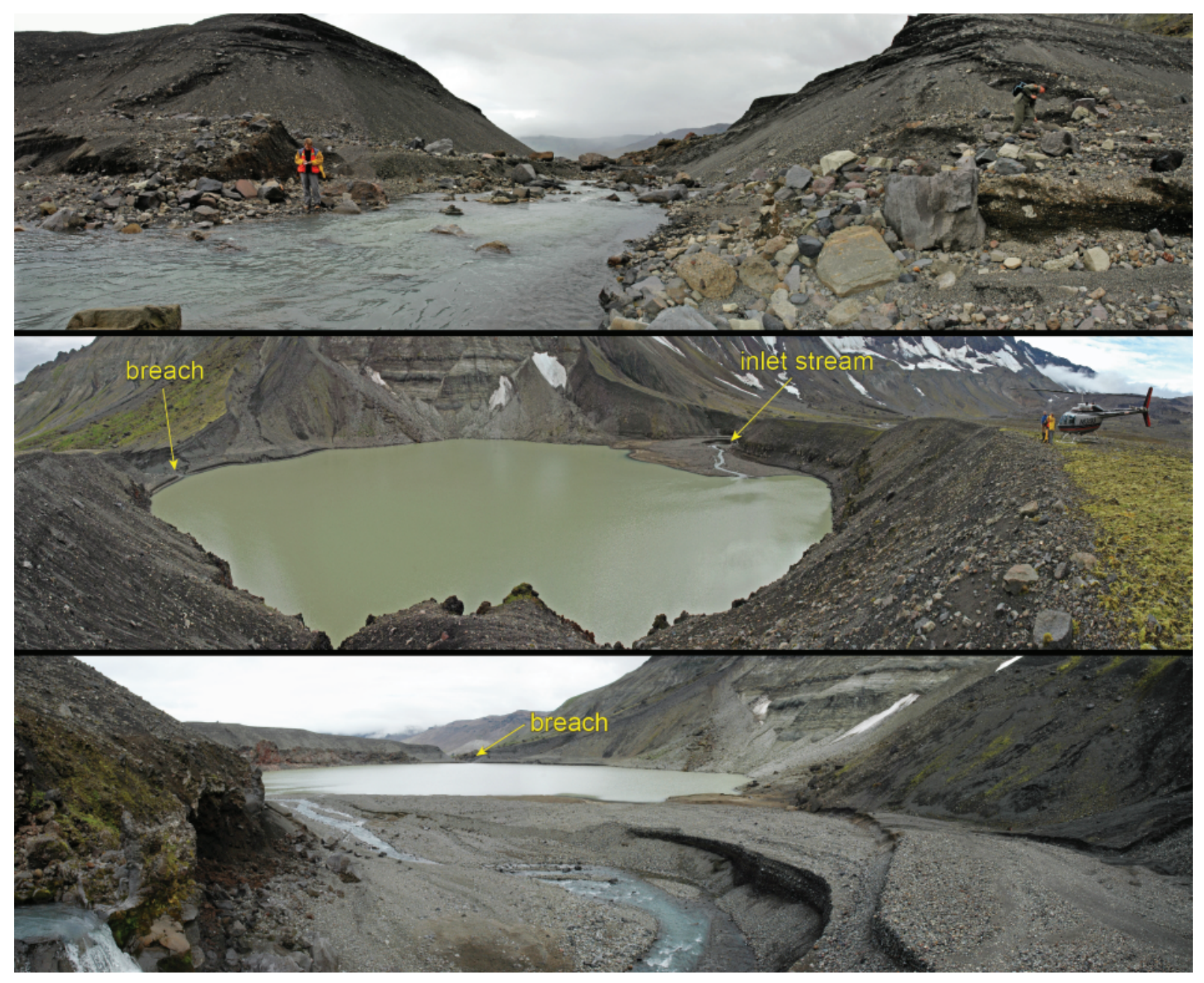

Figure 9. Panoramic views of the maar lake. Top: Breach through the northern rim of the maar crater lake; geologist Tina Neal (left) and NPS Resource Manager Troy Hamon (right) for scale. The lake level prior to the flood event was at least $4.65 \mathrm{~m}(15.3 \mathrm{ft}$ ) above the stream where Tina Neal is standing in this view. Center: Maar lake viewed from the western rim showing both the inlet stream and breach. Bottom: View north from the inlet stream across the incised delta of sediments and the maar lake. Images were taken by Game McGimsey, AVO/USGS, September 7, 2011. AVO database images at URL: http://www.avo.alaska.edu/images/image.php?id=57651 (top); $\underline{\text { http://www.avo.alaska.edu/ }}$ images/image.php?id=57661 (center); http://www.avo. alaska.edu/images/image.php?id=57681 (bottom). 


\section{Okmok Volcano}

CAVW\# 1101-29-

$53^{\circ} 25^{\prime} \mathrm{N} 168^{\circ} 08^{\prime} \mathrm{W}$

$1,073 \mathrm{~m}(3,502 \mathrm{ft})$

Umnak Island, eastern Aleutian Islands

INFLATION OF CALDERA FLOOR CONTINUES

Okmok volcano had a dramatic phreatomagmatic eruption over a 5-week period during the summer of 2008 (Neal and others, 2011). The eruption ended in August 2008 with seismic activity returning to background levels and remaining there until the morning of March 2, 2009, when a series of strong tremor-like events were recorded over a 24-hour period. Deformation (uplift) of the caldera floor began in August 2008 as the eruption ended and campaign GPS data from September 1, 2008, to March 1, 2009, showed a linear progression of $9 \mathrm{~cm}$ (3.5 in.) of uplift in the center of the caldera (see McGimsey and others, 2014). This rapid inflation slowed somewhat by the middle of 2010. In 2011, the inflation at Okmok continued, increasing over recent (2010) rates (5-6 cm over a 12-month period ending in September 2011), but still at a lower rate than was recorded following the 2008 eruption (M. Kaufman, UAFGI, written commun., September 7, 2011). In summary, the inflation at Okmok continues - albeit in pulses - with rates in the 5-7 cm/yr range (J. Freymueller, UAFGI, written commun., September 28, 2013).
Okmok Volcano is a $10-\mathrm{km}$-wide (6.2-mi) caldera that occupies most of the eastern end of Umnak Island, located $120 \mathrm{~km}(75 \mathrm{mi})$ southwest of the important fishing and transportation hub of Unalaska/Dutch Harbor in the eastern Aleutian Islands. Okmok has had several historic eruptions typically consisting of ash emissions occasionally more than $9 \mathrm{~km}(30,000 \mathrm{ft}) \mathrm{ASL}$, but generally much lower; lava flows crossed the caldera floor in 1945, 1958, and 1997 (Begét and others, 2005). The nearest settlement is Nikolski, $72 \mathrm{~km}$ (45 $\mathrm{mi}$ ) west of the volcano, with a population of 27 (Alaska Community Database, Community Information Summaries: http:/www.commerce.state.ak.us/dca/commdb/CIS.cfm; accessed November 14, 2012). A ranch caretaker family lives at Fort Glenn on the flank of the volcano about $10 \mathrm{~km}(6.2 \mathrm{mi})$ east of the caldera rim. 


\section{Cleveland Volcano}

CAVW\# 1101-24-

$52^{\circ} 49^{\prime} \mathrm{N} 169^{\circ} 57^{\prime} \mathrm{W}$

$1,730 \mathrm{~m}(5,676 \mathrm{ft})$

Chuginadak Island, east-central Aleutian Islands

THERMAL ANOMALIES, LAVA DOME EXTRUSION, AND MINOR ASH PLUMES

Ash clouds, thermal anomalies, lava dome growth, fumarole steaming, and ballistics observed

Cleveland volcano remained at Aviation Color Code YELLOW and Volcano Alert Level ADVISORY throughout the first 3 months of 2011. In February, an eyewitness pilot report (PIREP) described Mount Cleveland's summit as lightly steaming, with no visible signs of recent activity present on the volcano's upper flanks. On March 31, AVO downgraded the Aviation Color Code/Volcano Alert Level from YELLOW/ADVISORY to UNASSIGNED following the continued absence of thermal anomalies at Cleveland in the twice-daily AVO satellite monitoring shifts. Cleveland remained UNASSIGNED from March 31 to July 20. On July 20, AVO upgraded the Aviation Color Code/Volcano Alert Level from UNASSIGNED to YELLOW/ADVISORY after thermal anomalies were observed in satellite imagery during routine satellite monitoring on July 16-17 (ig. 10). On August 2, the Aviation Color Code/Volcano Alert Level was upgraded to ORANGE/WATCH based on persistent thermal anomalies detected at the volcano's summit (fig. 11) as well as satellite evidence of new lava in the summit crater on July 31 .

In 2011, Cleveland's summit crater was about 200-225 m (660-740 ft) wide at the rim; its depth varies through time with the impacts of eruptive activity, but can be as much as $80-100 \mathrm{~m}(260-330 \mathrm{ft})$. Extrusion of lava in the summit crater presumably began around the time of the onset of persistent thermal anomalies, during July 16-17; a new dome in late July 2011 was approximately $40 \mathrm{~m}$ (130 ft) across. Satellite images of the summit on August 3 showed the dome to be approximately $50 \mathrm{~m}(160 \mathrm{ft})$ across and no more than $20 \mathrm{~m}$ (65 ft) above the summit crater floor. The dome may

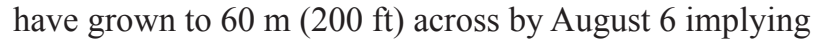
an approximate lava volume of $115,000 \mathrm{~m}^{3}\left(150,420 \mathrm{yds}^{3}\right)$ or 7 percent of the crater's total volume of approximately 1.6 million m3 (2.1 million yds3). Satellite imagery (fig. 12) showed no significant new tephra deposits indicating that activity from mid-July into early August was primarily extrusive. This was consistent with an August 9 WorldView-1 satellite image of Cleveland's summit showing steaming, light-colored alteration deep inside the summit crater around the new lava dome (fig. 13), and with oblique aerial photographs taken on August 8 by NOAA scientists (fig. 14).
On August 10, AVO received a mariners report from the National Weather Service (NWS) Ocean Prediction Center of possible ash floating on the sea surface approximately $25 \mathrm{~km}$ $(13.5 \mathrm{nmi})$ north-northwest of Cleveland. The same source reported the absence of any floating ash earlier in the day at about $30 \mathrm{~km}(16 \mathrm{nmi})$ north of the volcano. No ash clouds were detected in satellite data during the times of these reports. Analysis of satellite data indicated that during August 6-13, the lava dome grew only slightly larger than detected in the previous image acquired on August 6. Although it is possible that these accounts of drifting ash are valid, AVO was unable to confirm them.

On August 30, AVO downgraded Cleveland's Aviation Color Code/Volcano Alert Level from ORANGE/WATCH to YELLOW/ADVISORY based on the absence of distinct thermal signals at the summit. Satellite observations on September 6 indicated that the lava dome had grown to about $120 \mathrm{~m}$ (390 ft) in diameter and consistently elevated surface temperatures were again observed. As a result of these observations, AVO upgraded Cleveland to Aviation Color Code/Volcano Alert Level ORANGE/WATCH. By this time, the lava dome essentially filled the summit crater.

TerraSAR-X satellite radar images from the German Remote Sensing Data Center (DFD) and the German Aerospace Center (DLR), acquired over Cleveland volcano from August to November 2011, provided an image time series showing the partial growth of the 2011 lava dome in the summit crater (fig. 15).

The lava dome continued to grow through late September, expanding in diameter from approximately $120 \mathrm{~m}$ (390 ft) on September 6 to approximately $168 \mathrm{~m}(550 \mathrm{ft})$ by September 20 and reaching a height approximately $15-20 \mathrm{~m}$ (50-65 ft) below the crater rim by September 26. Additional growth of the dome past October 20 was minor (fig. 15).

Extrusion of lava either slowed or ceased between October 1 and October 5. Satellite data from October 9 indicated that the central portion of the lava dome became slightly depressed, indicating minor deflation of the dome. Subsidence of the dome continued into late October. 


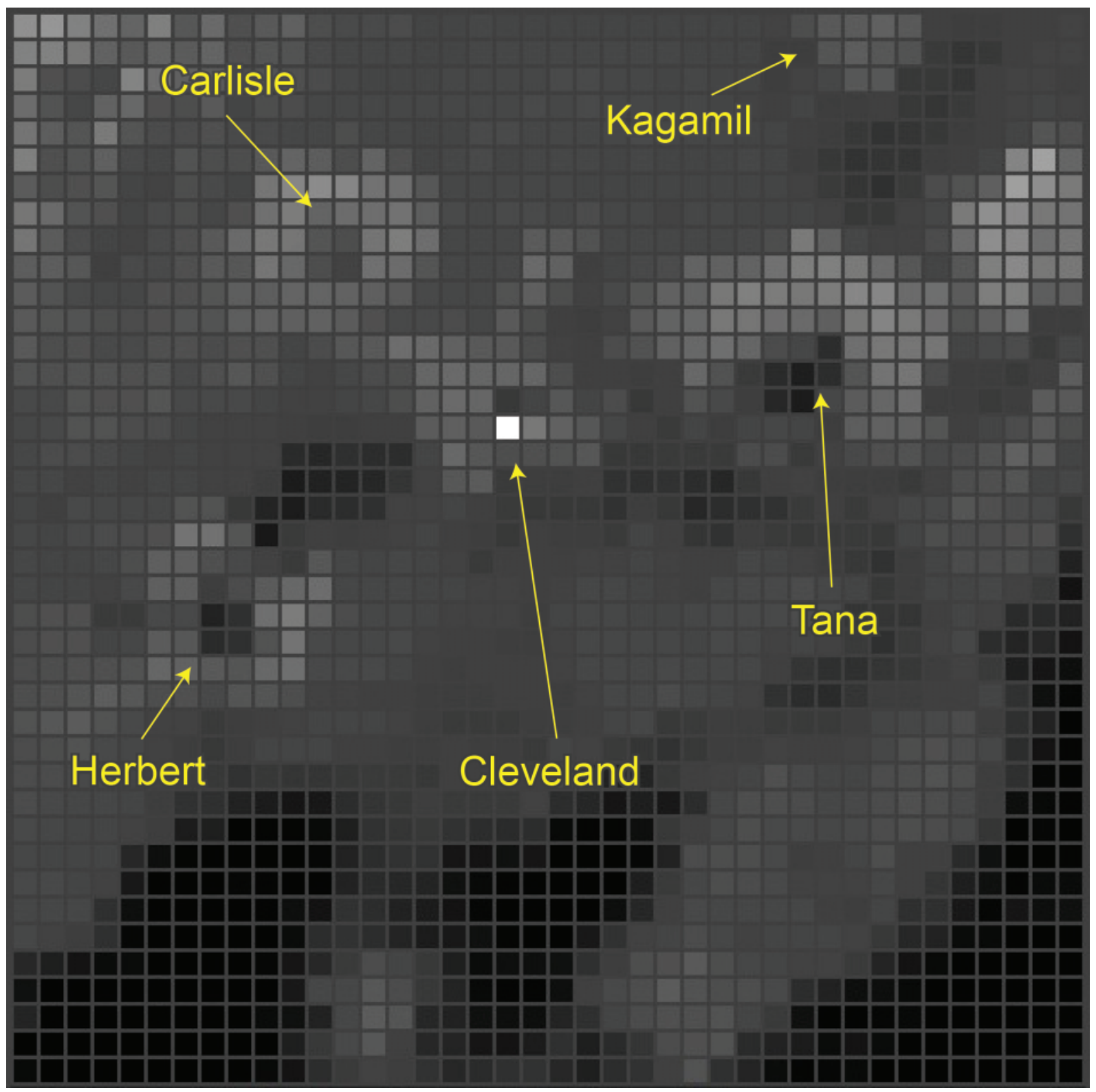

Figure 10. Elevated temperature anomaly (white pixel) at the summit of Cleveland volcano in a NOAA Advanced Very High Resolution Radiometer (AVHRR) satellite image, channel 3 (thermal band) from 06:12 UTC July 17, 2011. This screen shot is from output generated by the University of Alaska Fairbanks Geophysical Institute's Remote sensing group's Hotspot Viewer tool. Outlines of the islands of Carlisle, Herbert, and Kagamil are faintly visible; Cleveland and Tana volcanoes comprise Chuginadak Island. 


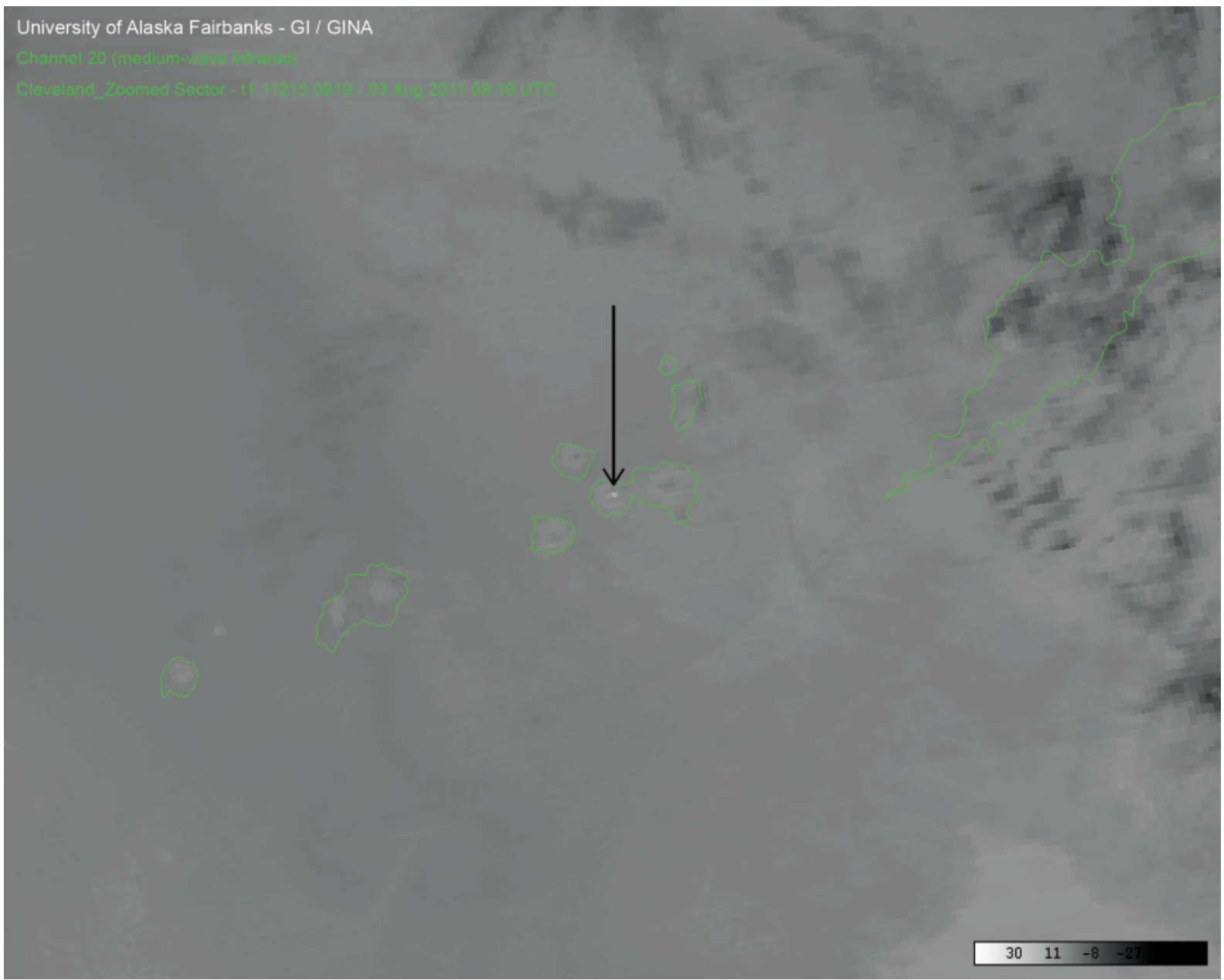

Figure 11. MODIS satellite thermal image (250-m resolution) of the Islands of the Four Mountains and Umnak Island in the eastern Aleutians on August 3, 2011. An area of elevated temperature at the summit of Cleveland volcano (arrow) was consistent with presence of lava at the surface. Such thermal signals are often masked by heavy cloud cover in the Aleutians. Image courtesy Peter Webley, UAFGI. 


\section{Cleveland Volcano, Alaska 7 August 2011 Landsat ETM+15m pan image}

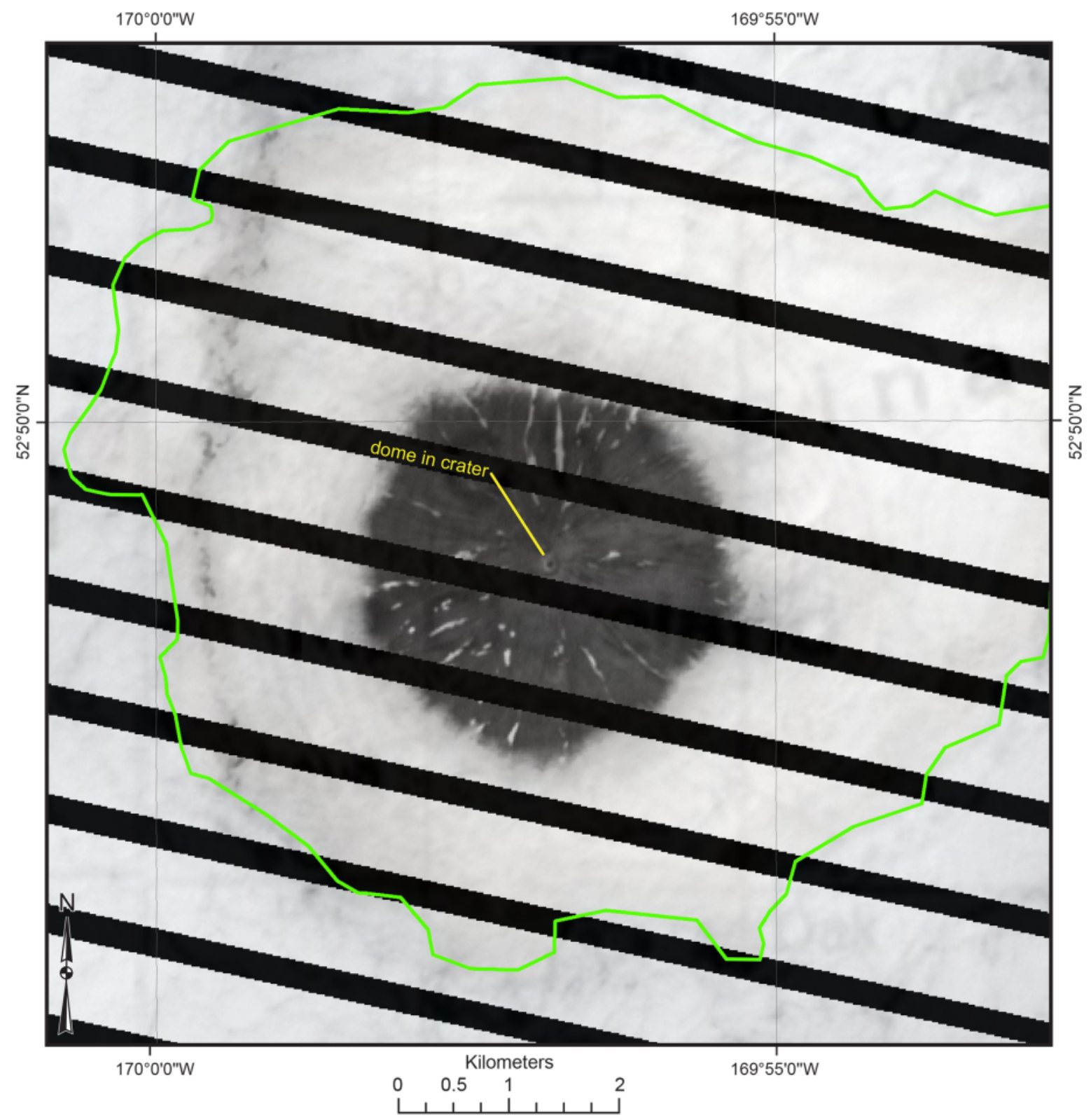

Figure 12. Landsat images of Cleveland volcano from August 7, 2011. (A) Landsat ETM+15m panchromatic band. $(B)$ Landsat ETM+30m multi-spectral. Green line is the outline of the southwestern part of Chuginadak Island. Red pixel in multi-spectral image $(B)$ shows elevated temperatures of the lava dome within the summit crater. Diagonal black lines are data gaps. Images courtesy Rick Wessels, USGS. 


\section{Cleveland Volcano, Alaska 7 August 2011 Landsat ETM+30m b742RGB image}

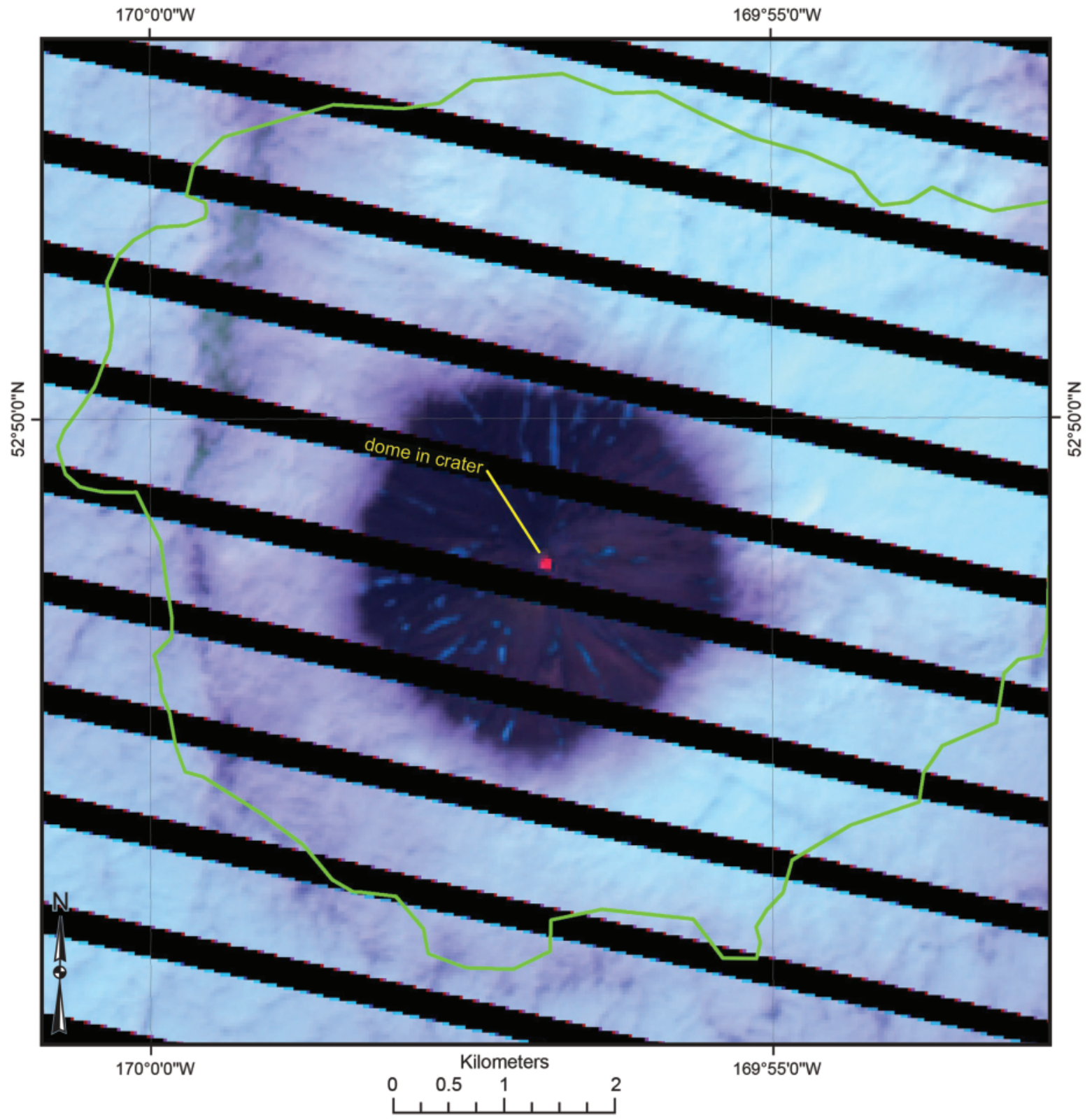

Figure 12.-Continued 


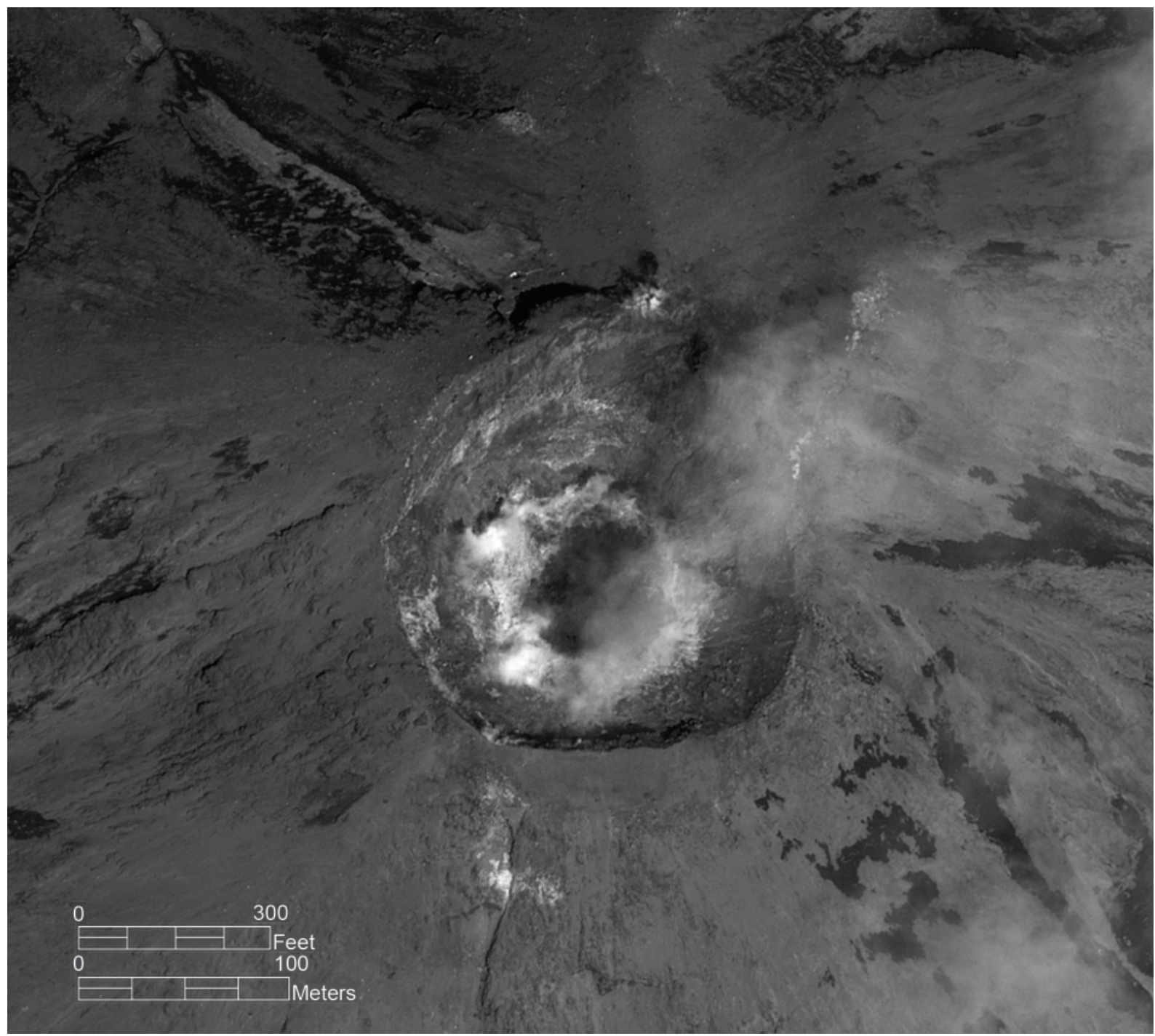

Figure 13. WorldView-1 satellite image of the summit of Cleveland volcano, August 9, 2011. 

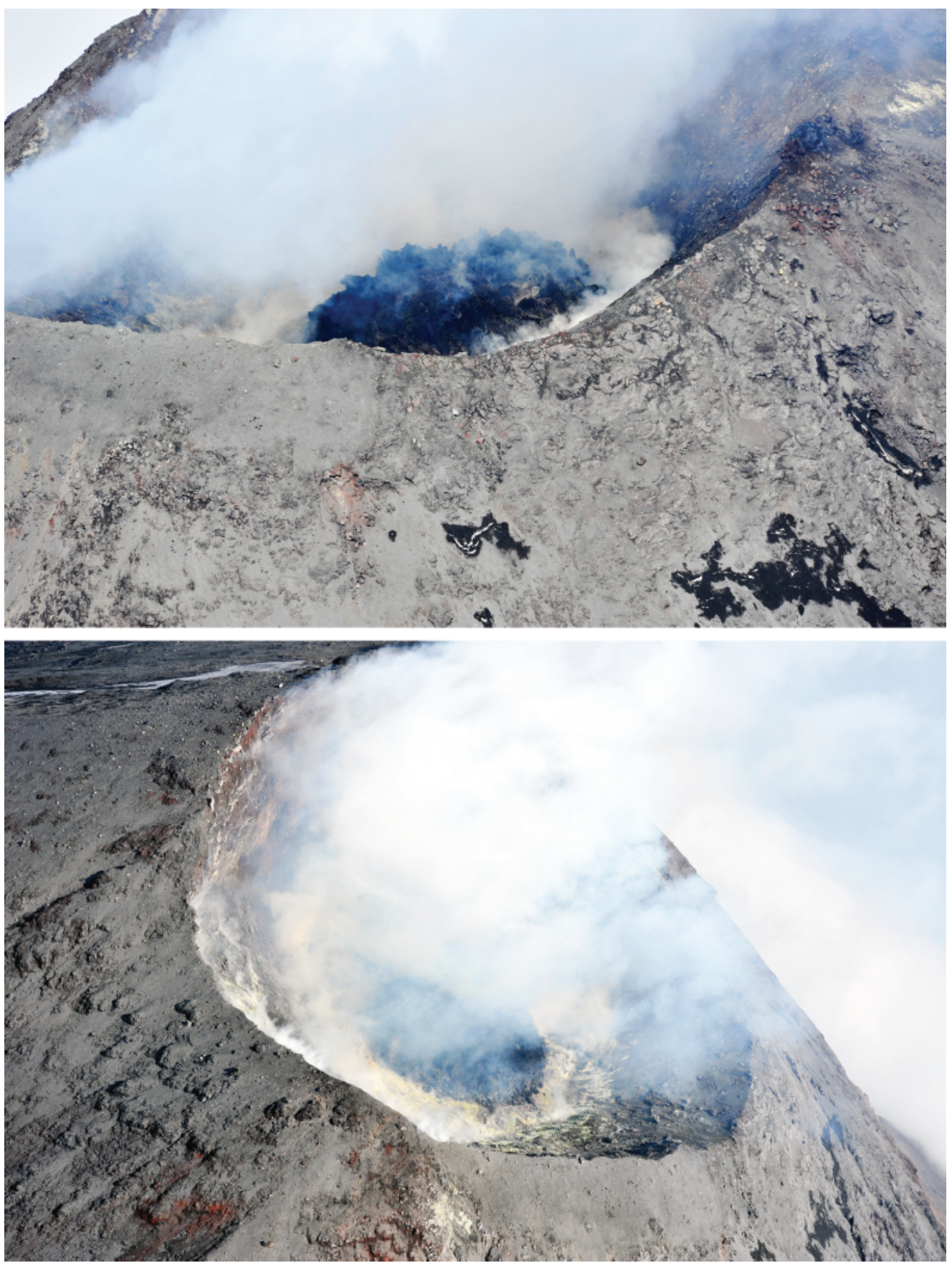

Figure 14. Oblique aerial photographs of the summit crater of Cleveland volcano on August 8, 2011. (A) Fume billows from the growing lava dome erupting onto the floor of the crater. At this time, the dome was at least $60 \mathrm{~m}(200 \mathrm{ft}$ ) in diameter. $(B)$ View of the dome inside the 200 to $225 \mathrm{~m}$ (660 to $740 \mathrm{ft}$ ) diameter crater filled with fume. Note the light colored deposits on the crater floor and walls around the dome, presumably sulfur and other precipitates. In both images, the upper flanks of the cone are mantled by recently erupted gray tephra. Photographs courtesy Dave Withrow, NOAA. AVO database image (A) at URL: http://www.avo.alaska.edu/images/image.php?id=36322. AVO database image (B) at URL: http://www.avo.alaska.edu/images/image.php?id=36422. 

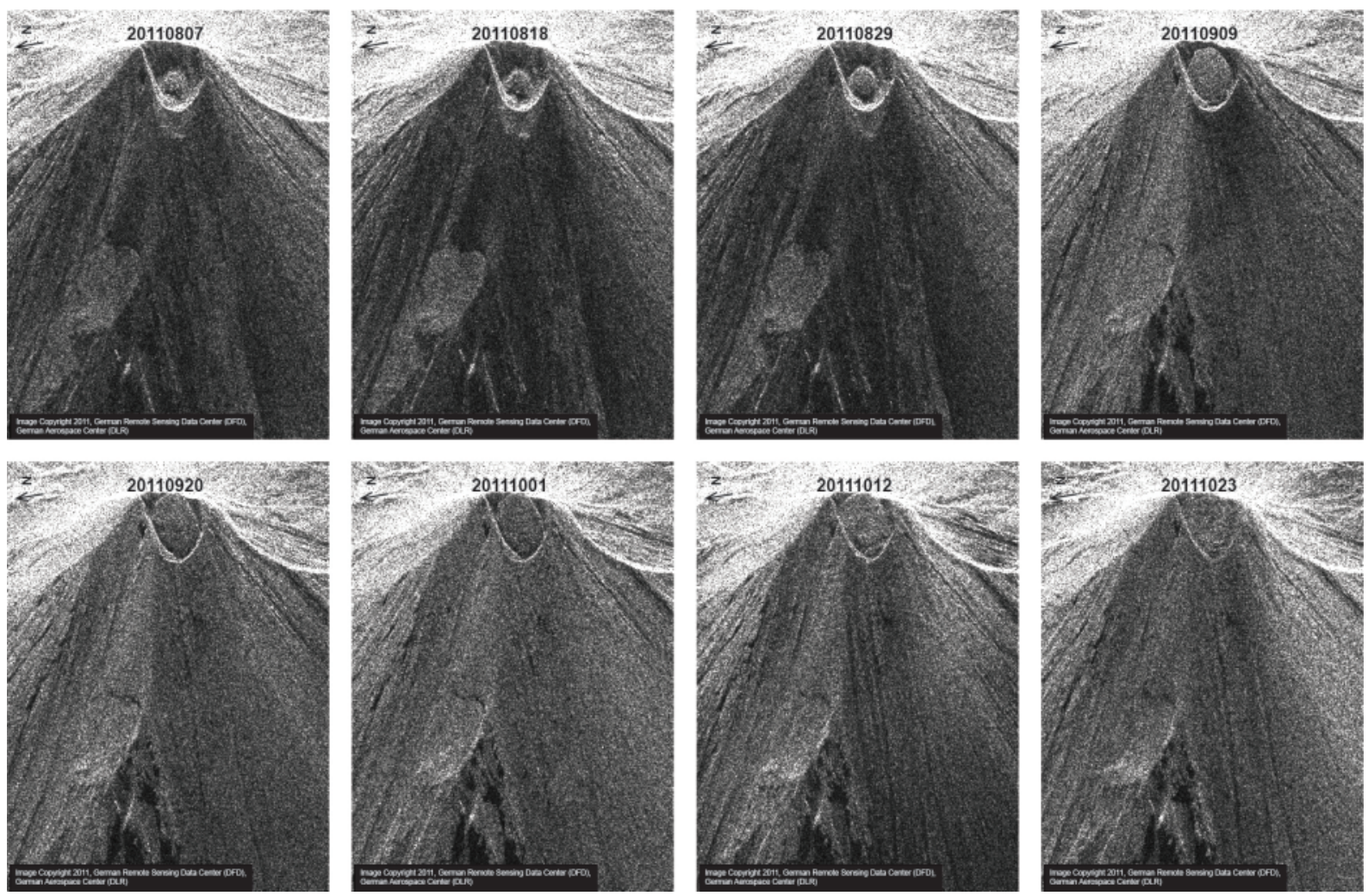

Figure 15. Time sequence of TerraSAR-X images of the Cleveland cone and summit crater from August 7 to 0 ctober 23, 2011, gathered by Zhong Lu of the USGS Cascade Volcano Observatory. Successive panels show the growth of the lava dome as it filled the 200-225 m (660-740 ft) diameter summit crater nearing the elevation of the eastern rim (see last panel). North is to the left in all images. Images are copyright German Remote Sensing Data Center (DFD), German Aerospace Center (DLR).

AVO downgraded Cleveland's Aviation Color Code/ Volcano Alert Level from ORANGE/WATCH to YELLOW/ ADVISORY on November 3 based on the absence of consistent thermal anomalies in satellite images and apparent cessation of lava effusion after October 9.

On November 10, satellite images showed that a small secondary dome had emerged atop the center of the semideflated lava dome. The diameter of this new dome was approximately $15-20 \mathrm{~m}(50-65 \mathrm{ft})$ and it likely began to grow on or before November 2 . The original dome remained unchanged in size.

Lava within the summit crater remained mostly unchanged from November 10 to November 24. A satellite image from November 25 showed that the small secondary dome had subsided into a broad blocky, hummocky depression approximately $70 \mathrm{~m}(230 \mathrm{ft})$ in diameter and the overall dome had subsided approximately $30-35 \mathrm{~m}(100-115 \mathrm{ft})$ from its maximum elevation in early October. The dome continued to subside into early December, and by December 7, nearly the entire extrusive feature had collapsed into the conduit and its surface was approaching the pre-August crater-floor elevation.
On December 29 at approximately 04:12:07 AKST (13:12:07 UTC), an explosion from Cleveland produced a small ash cloud that rose to approximately $3.5 \mathrm{~km}$ $(11,500 \mathrm{ft}) \mathrm{ASL}$. The ash cloud drifted to the east and over the southwestern tip of Umnak Island (fig. 16). The eruption triggered two operational ash alarms used by AVO. The first alarm was triggered at approximately 05:33 AKST (14:33 UTC) indicating likely ash signatures in NOAA's AVHRR satellite image n19.11363.1402 (ig. 16) A NOAANESDIS ash cloud alarm was triggered at approximately 05:34 AKST (14:34 UTC) from the same AVHRR satellite image. Calculations based on the satellite data and local meteorological conditions indicated a maximum ash cloud height of $3.5 \mathrm{~km}(11,500 \mathrm{ft})$ ASL, with a mean effective ash particle radius of 5.06 microns $\left(1.99 \times 10^{-4} \mathrm{in}\right.$.), a total mass of $0.84 \mathrm{kt}$ (925 tons), and a total area of $173 \mathrm{~km}^{2}\left(66.8 \mathrm{mi}^{2}\right)$ (M. Pavolonis, written commun., December 29, 2011).

In response to the ash cloud, AVO upgraded the Aviation Color Code/Volcano Alert Level from YELLOW/ ADVISORY to ORANGE/WATCH at 07:55 AKST (16:55 UTC) on December 29, 2011. 


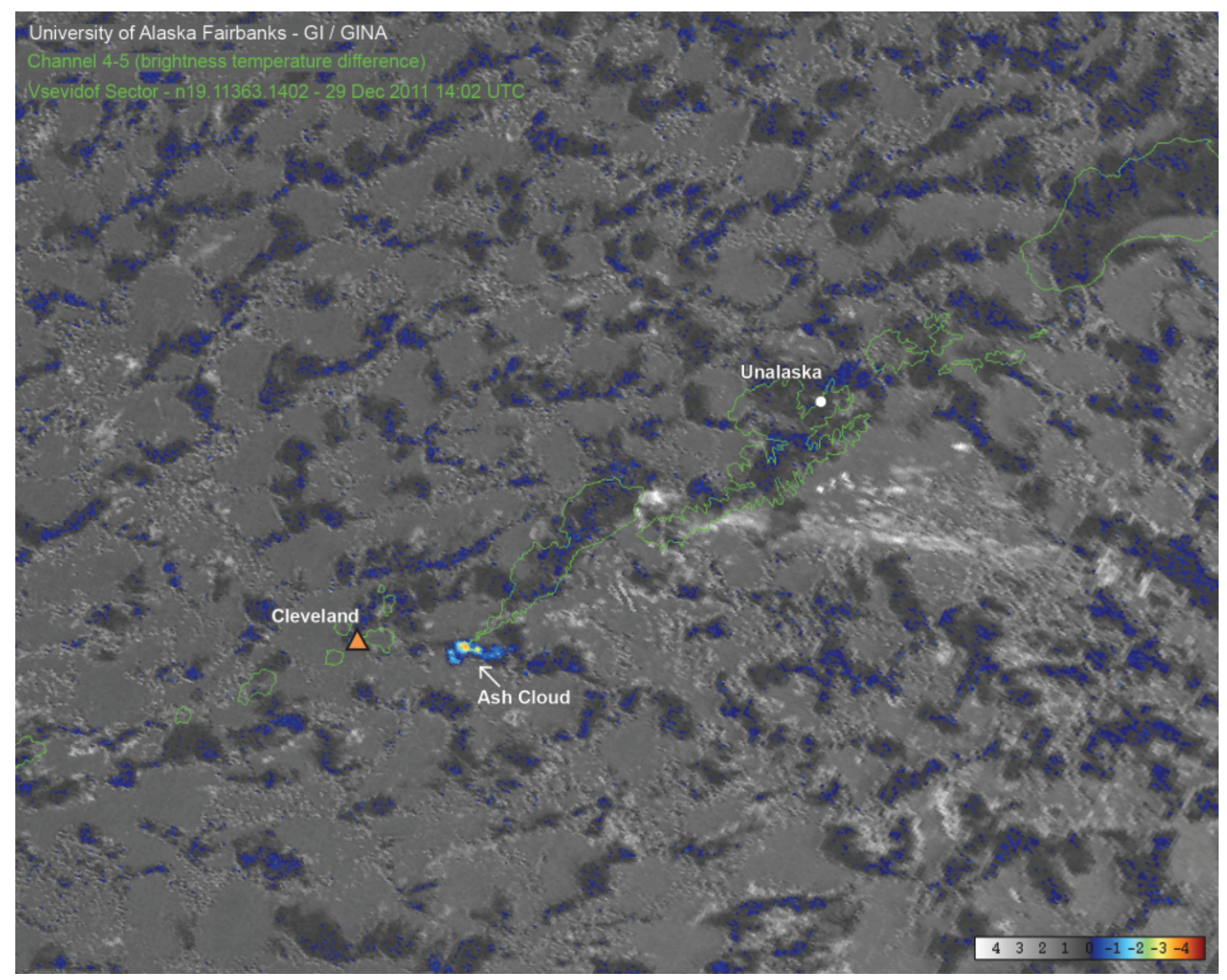

Figure 16. NOAA AVHRR satellite image from 14:02 UTC on December 29, 2011. The brightly colored region indicated ash in the atmosphere over the southwestern tip of Umnak Island. This is a brightness temperature difference image (BTD). Satellite data provided by UAFGI/GINA.

Infrasound signals from the December 29 explosion were detected on seismic stations and infrasound arrays deployed at Okmok volcano, located approximately $139 \mathrm{~km}$ (90 mi) northeast of Cleveland on Umnak Island (fig. 17). Infrasonic waves are sound waves that span a frequency range from below 20 Hertz (the lower limit of human hearing) to $0.001 \mathrm{Hertz}$. Infrasound signals are recorded at seismic stations by the infrasound airwaves coupling with the ground at seismic station(s) and mechanically vibrating the ground in which the seismometer sits. The recorded seismic signal is known as a ground-coupled airwave produced by a volcanic explosion or eruption. Based on the speed of sound in Earth's atmosphere and the distance between seismic station OKWE and the summit of Cleveland the origin time of the explosion was calculated at approximately 04:12:07 AKDT
(13:12:07 UTC) (Matt Haney, David Fee, and Silvio de Angelis, UAFGI, written commun., December 29, 2011).

AVO downgraded the Aviation Color Code/Volcano Alert Level for Cleveland volcano from ORANGE/WATCH to YELLOW/ADVISORY at 13:57 AKST (22:57 UTC) on December 29 following no additional reports of eruptive activity occurring at the volcano. Cleveland remained at Aviation Color Code/Volcano Alert Level YELLOW/

ADVISORY throughout the remainder of 2011.

A review of the infrasound data prior to the December 29 explosion revealed several small explosive eruptions from Cleveland volcano on December 25. The first occurred at approximately 03:13 AKST (12:13 UTC) and had an infrasound amplitude of approximately one-half the December 29 event. The second eruption occurred on December 25 at approximately 06:32 AKST (15:32 UTC). 

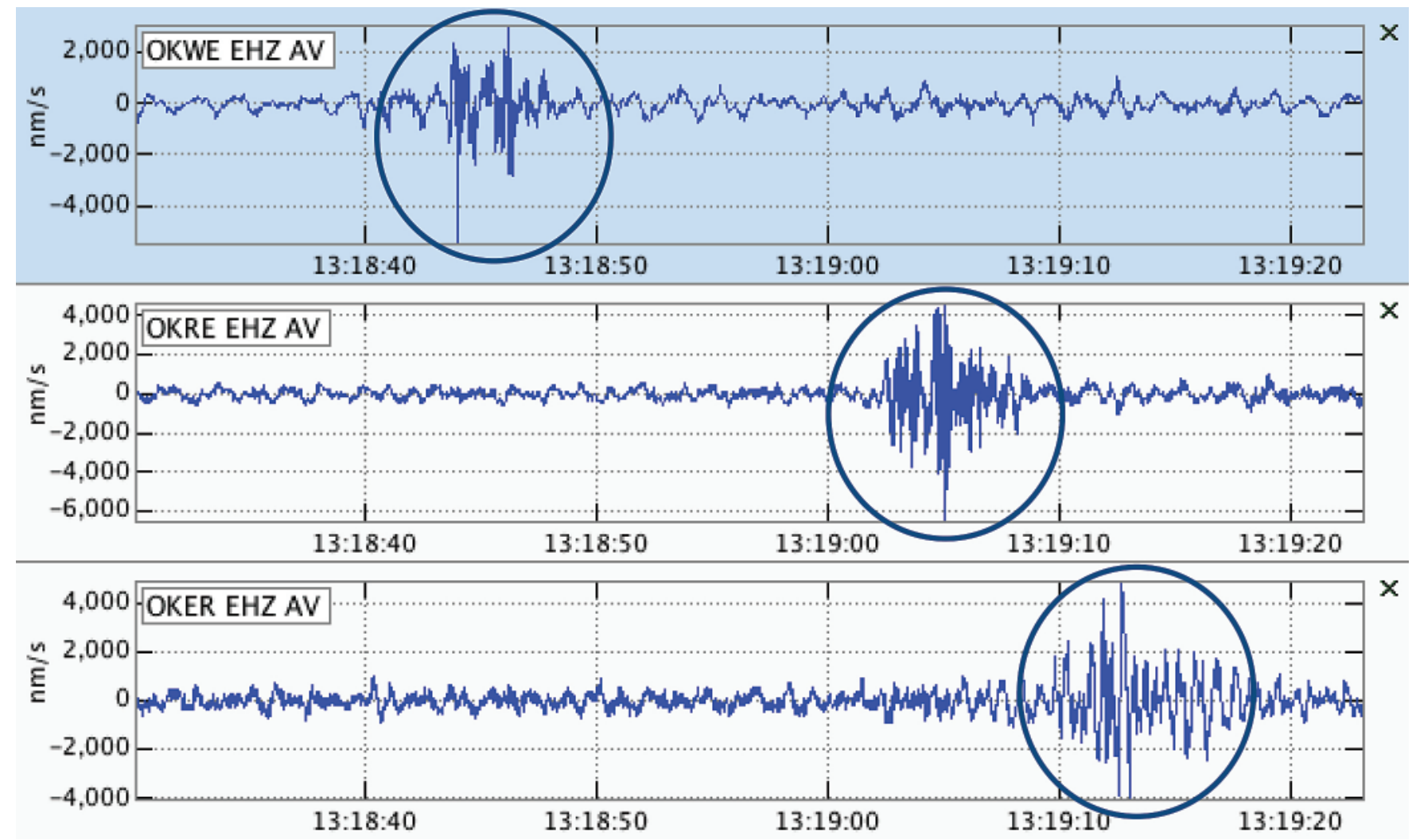

Figure 17. Record of the December 29, 2011, explosion at Cleveland volcano on seismic stations OKWE, OKRE, and OKER (circles). Image courtesy Matt Haney, AVO/USGS.

A small ash cloud also was retrospectively detected in satellite imagery for the December 25 event. The eruption cloud was very minor, did not have a large ash signal at the image's collection time of 06:32 AKST (15:32 UTC), and was only weakly visible in a thermal infrared image. The cloud had dissipated by the time the next image was acquired at 06:46 AKST (15:46 UTC).

Satellite data from December 26 displayed evidence of ejected blocks that had rolled down the upper northern and western flanks of the volcano, some as far as about 1.5 $\mathrm{km}(5,000 \mathrm{ft})$ from the crater's rim. There was no indication of fresh ash deposits on the volcano's upper northern and western flanks.

As in previous years, AVO tracked and responded to Cleveland activity in 2011 by relying heavily on satellite data and to reports received from pilots or other sources. AVO has no seismic instruments located on Chuginadak Island, therefore monitoring of Cleveland volcano is accomplished by analyzing daily satellite images, occasional pilot and mariner reports, and on rare clear days, a Web camera located in Nikolski. Table 5 is a compilation of Aviation Color Code and Volcano Alert Level changes, activity, ground and air observations of ash clouds, elevated surface temperatures, other satellite observations, and seismic network and infrasound detection or other alarm triggers.

Mount Cleveland volcano forms the western part of Chuginadak Island, an uninhabited island in the Islands of Four Mountains group in the east-central Aleutians. Cleveland is located about $75 \mathrm{~km}$ (45 mi) west of the community of Nikolski, and 1,500 km (940 mi) southwest of Anchorage. Historical eruptions have been characterized by short-lived ash explosions, lava fountaining, lava flows, and pyroclastic avalanches down the flanks. In February 2001, after 6 years of quiescence, Cleveland had three explosive events that produced ash clouds as high as $12 \mathrm{~km}(39,000 \mathrm{ft})$ ASL (Dean and others, 2004), a rubbly lava flow, and a hot avalanche that reached the sea. Intermittent explosive eruptions have occurred in every year since 2001 . 


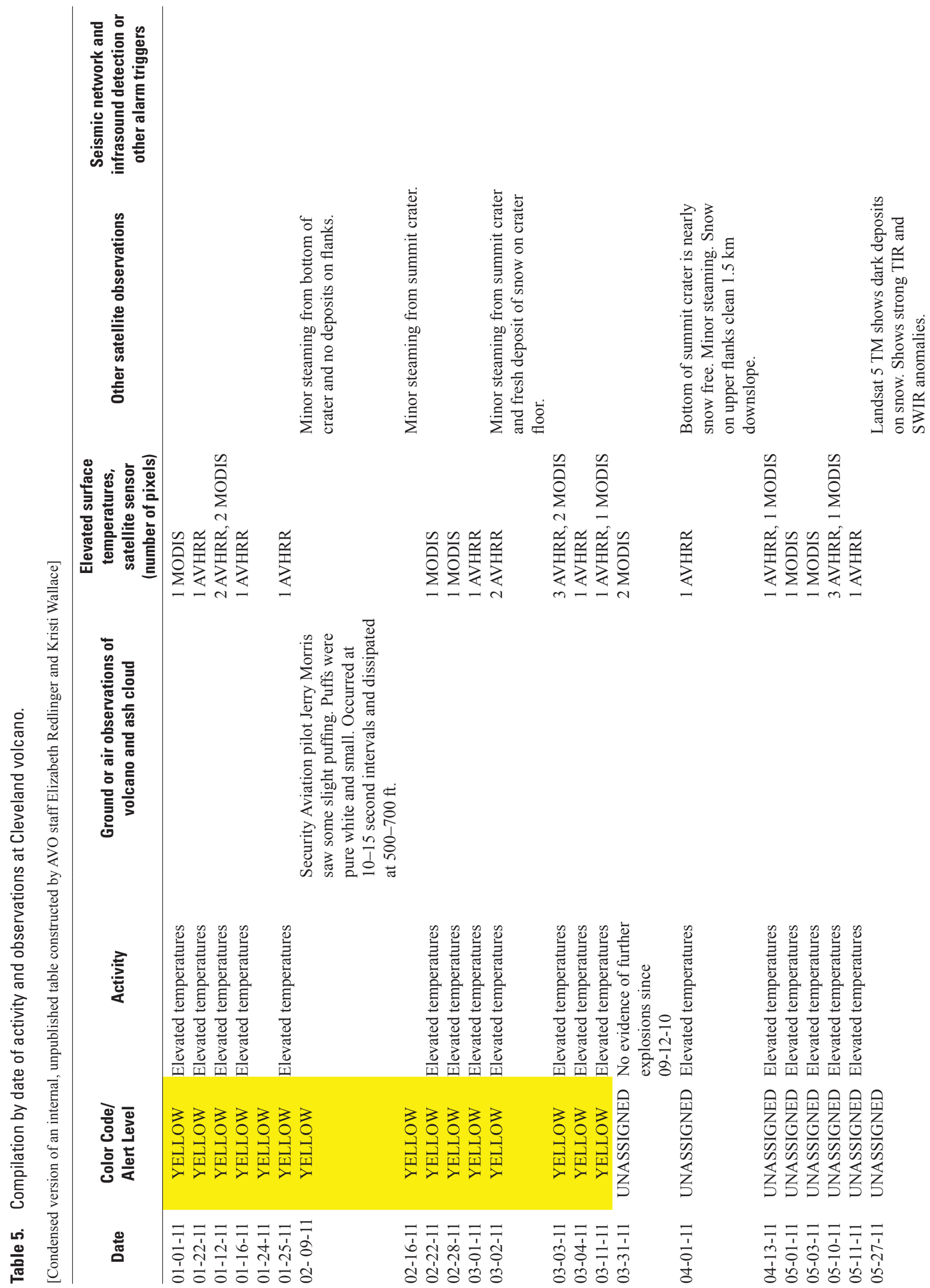




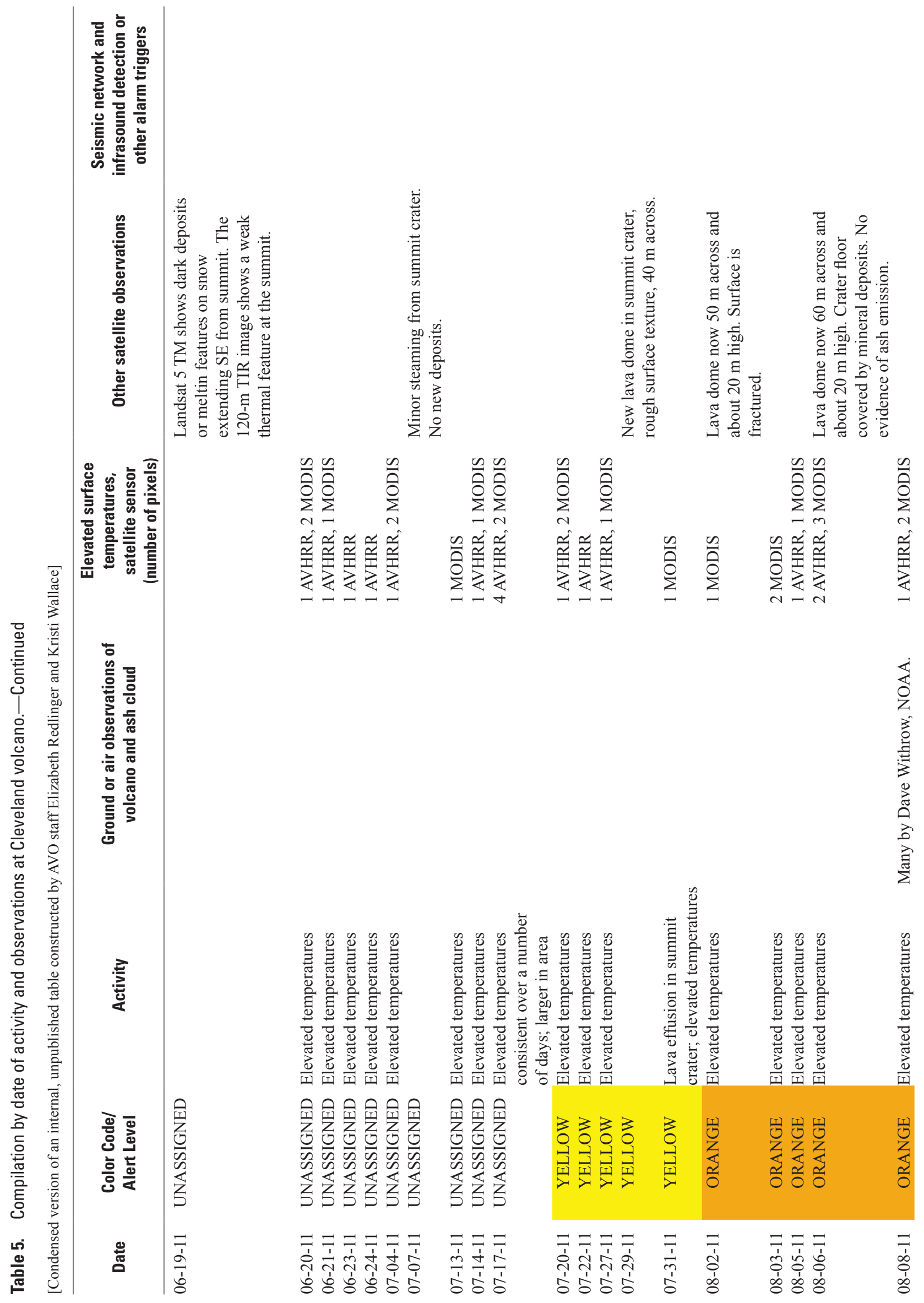




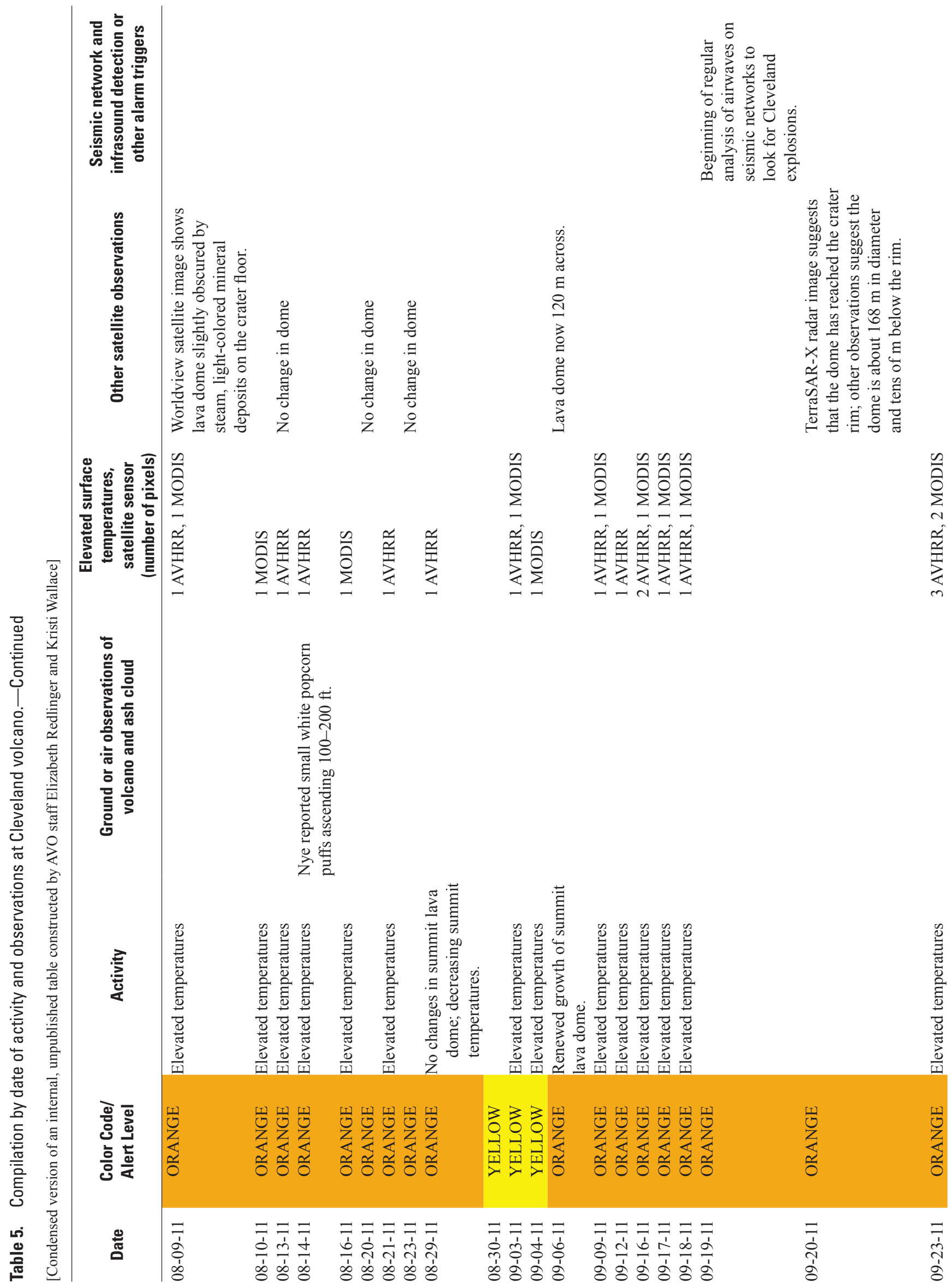




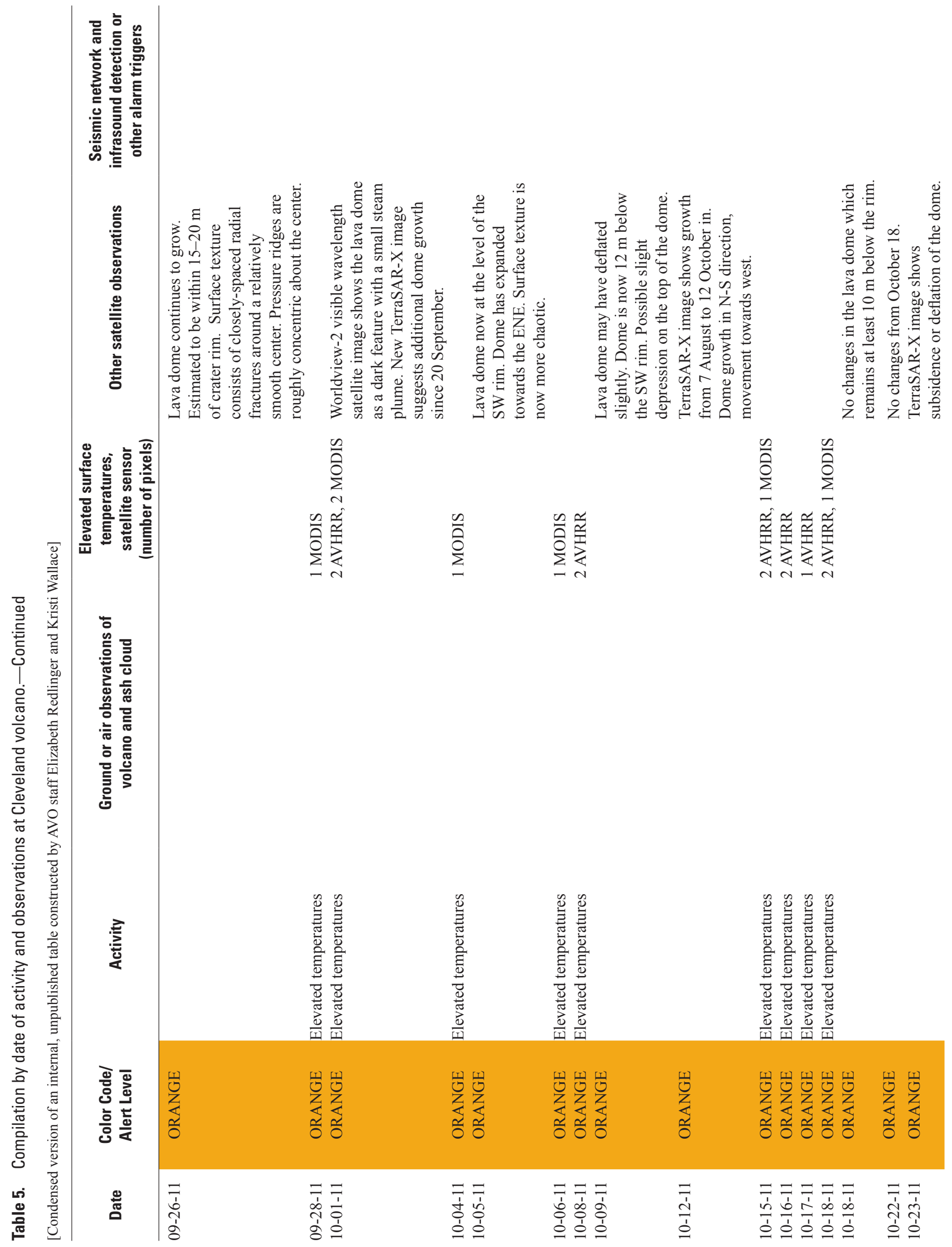




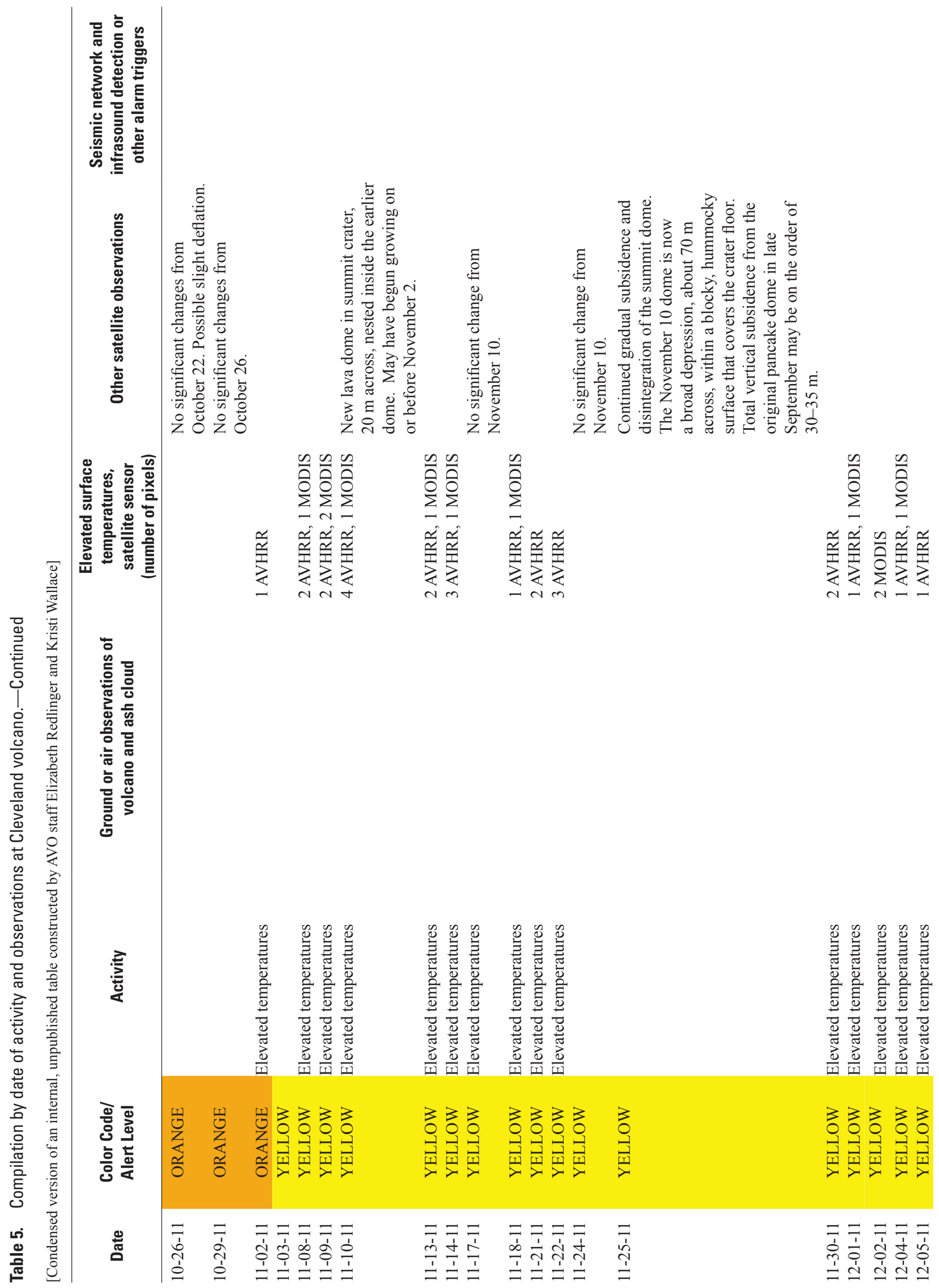




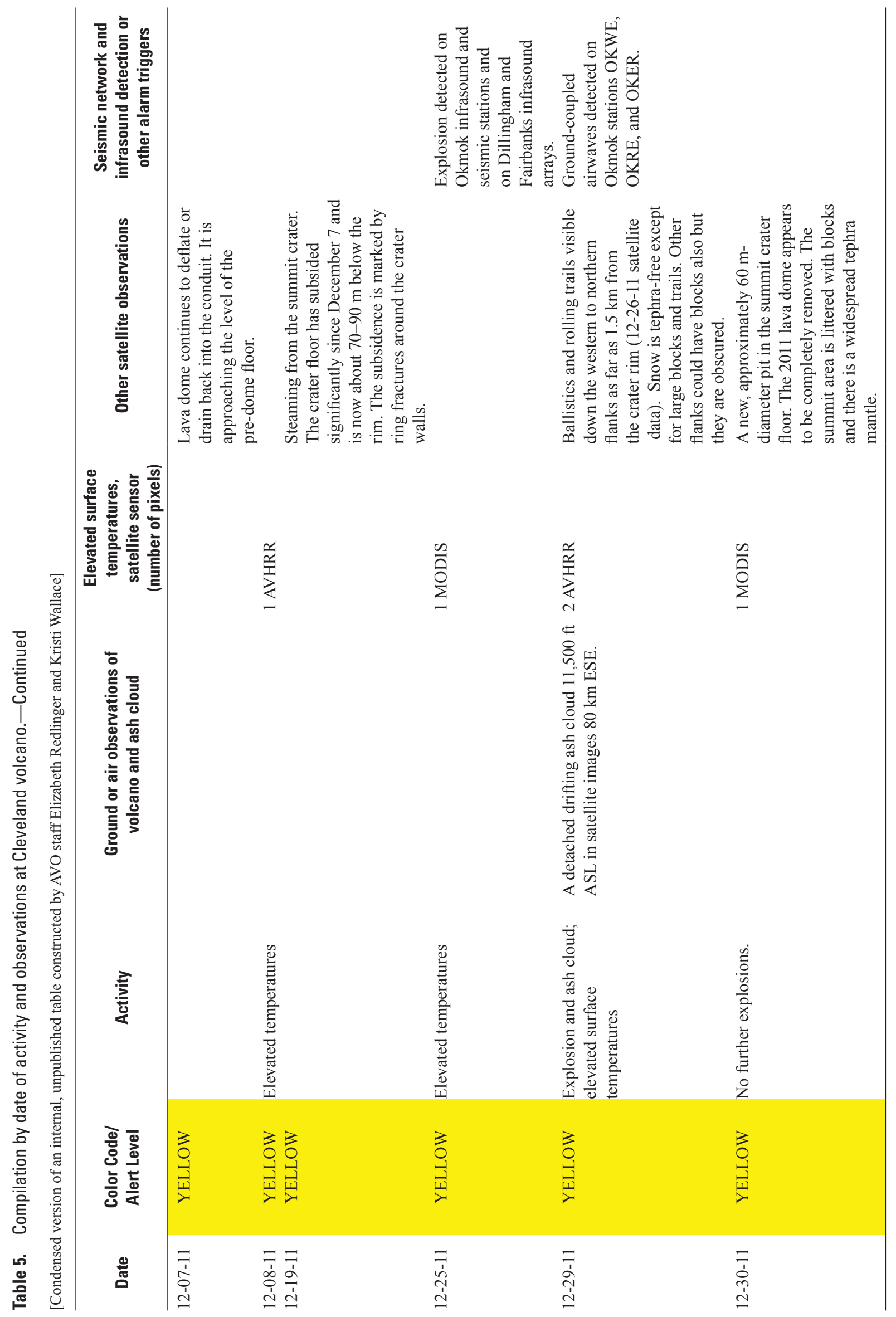




\section{Summary}

The Alaska Volcano Observatory (AVO) responded to volcanic unrest at three volcanic centers in Alaska, including an ongoing eruption from Cleveland, one of Alaska's most frequently active volcanoes. Relatively rapid inflation of the caldera floor at Okmok, which began immediately following the 2008 eruption, continued. Partial draining of the larger of two maar crater lakes at Aniakchak, which occurred in late summer 2010, was discovered and investigated in 2011. AVO annual summaries from 1995 to 2010 have included reports on activity at Russian volcanoes. In 2011, AVO ceased including Russian activity in the annual summary.

\section{Acknowledgments}

This report represents the work of the entire Alaska Volcano Observatory, colleagues from other USGS Volcano Observatories, staff at cooperating agencies, and the public. We also acknowledge the significant contributions of our colleagues at Alaska Division of Geological \& Geophysical Surveys for design and maintenance of the Alaska Volcano Observatory image database, a powerful tool for review of activity through the year. Technical reviews by Chris Waythomas and Sally Sennert improved the content and presentation. All images and photographs from our colleagues and the public in this report are appreciated and used with permission.

\section{Sources of Photographs in this Report and Other Images of Alaska}

\author{
Online sources of digital images from this report: \\ http://libraryphoto.cr.usgs.gov/ \\ http://www.avo.alaska.edu/images/ \\ http://pubs.usgs.gov/dds/dds-39/ \\ http://pubs.usgs.gov/dds/dds-40/
}

\section{References Cited}

Beget, J.E., Larsen, J.F., Neal, C.A., Nye, C.J., and Schaefer, J.R., 2005, Preliminary volcano hazard assessment for Okmok Volcano, Umnak Island, Alaska: Alaska Division of Geological \& Geophysical Surveys Report of Investigation 2004-3, 32 p., 1 sheet, scale 1:150,000.
Dean, K.G., Dehn, Jonathan, Papp, K.R., Smith, Steve, Izbekov, Pavel, Peterson, Rorik, Kearney, Courtney, and Steffke, Andrea, 2004, Integrated satellite observations of the 2001 eruption of Mt. Cleveland, Alaska: Journal of Volcanology and Geothermal Research, v. 135, p. 51-73.

Dixon, J.P., Stihler, S.D., Power, J.A., and Searcy, C.K., 2012, Catalog of earthquake hypocenters at Alaskan volcanoesJanuary 1 through December 31, 2011: U.S. Geological Survey Data Series 730, 82 p., http://pubs.usgs.gov/ds/730/ pdf/ds730.pdf.

Dreher, S.T., Eichelberger, J.C., and Larsen, J.F., 2005, The petrology and geochemistry of the Aniakchak calderaforming ignimbrite, Aleutian Arc, Alaska: Journal of Petrology, v. 46, no. 9, p. 1747-1768, doi:10.1093/ petrology/egi032.

Gardner, C.A., and Guffanti, M.C., 2006, U.S. Geological Survey's alert notification system for volcanic activity: U.S. Geological Survey Fact Sheet 2006-3139, 4 p., http:// pubs.usgs.gov/fs/2006/3139/.

Hubbard, B.R., 1931, A world inside a mountain-Aniakchak, the new volcanic wonderland of the Alaska Peninsula, is explored: National Geographic, v. 60, no. 3, p. 319-345.

Lu, Zhong, 2007, InSAR imaging of volcanic deformation over cloud-prone areas-Aleutian Islands: Photogrammetric Engineering and Remote Sensing, v. 73, no. 3, p. 245-257.

Lu, Zhong, Dzurisin, Daniel, Wicks, C.J., Power, John, Kwoun, O. and Rykhus, R., 2007, Diverse deformation patterns of Aleutian volcanoes from satellite interferometric synthetic aperture radar (InSAR), in Eichelberger, John. Gordeev, Evgenii, Kasahara, Minoru, Izbekov, Pavel, and Lees, Jonathan, eds., Volcanism and tectonics of the Kamchatka Peninsula and adjacent arcs, American Geophysical Union Monograph Series 172, p. 249-261.

Lu, Zhong, Wicks, Charles, Jr., Dzurisin, Daniel, Power, John, Thatcher, Wayne, and Masterlark, Timothy, 2003, Interferometric synthetic aperture radar studies of Alaska volcanoes: Earth Observation Magazine, v. 12, no. 3, p. $8-18$.

McGimsey, R.G., Neal, C.A, Girina, O.A., Chibisova, Marina, and Rybin, Alexander, 2014, 2009 Volcanic activity in Alaska, Kamchatka, and the Kurile Islands - Summary of events and response of the Alaska Volcano Observatory: U.S. Geological Survey Scientific Investigations Report 2013-5213, 125 p., http://dx.doi.org/10.3133/ sir20135213.

Miller, T.P., and Smith, R.L., 1987, Late Quaternary calderaforming eruptions in the eastern Aleutian arc, Alaska: Geology, v. 15, no. 5, p. 434-438. 
Neal, Christina, Girina, Olga, Senyukov, Sergey, Rybin, Alexander, Osiensky, Jeffrey, Izbekov, Pavel, and Ferguson, Gail, 2009, Russian eruption warning systems for aviation: Natural Hazards, v. 50, 18 p., doi:10.1007/s11069-0099347-6.

Neal, C.A., McGimsey, R.G., Dixon, J.P., Cameron, C.E., Nuzhdaev, A.A., and Chibisova, M., 2011, 2008 volcanic activity in Alaska, Kamchatka, and the Kurile Island-Summary of events and response of the Alaska Volcano Observatory: U.S. Geological Survey Scientific Investigations Report 2010-5243, 94 p., http://pubs.usgs. gov/sir/2010/5243.

Neal, C.A., McGimsey, R.G., Miller, T.P., Riehle, J.R., and Waythomas, C.F., 2001, Preliminary volcano-hazard assessment for Aniakchak Volcano, Alaska: U.S. Geological Survey Open-File Report 00-519, 35 p.
Nicholson, R.S., 2003, The 1931 eruption of Aniakchak Volcano, Alaska: University of Alaska Fairbanks, unpublished M.S. thesis, $270 \mathrm{p}$.

Riehle, J.R., Fleming, M.D., Molnia, B.F., Dover, J.H., Kelley, J.S., Miller, M.L., Plafker, George, and Till, A.B., 1997, Shaded relief image of Alaska: U.S. Geological Survey Miscellaneous Investigations Series MI-2585.

Schaefer, J.R., Cameron, C.E., and Nye, C.J., 2014, Historically active volcanoes of Alaska: Alaska Division of Geological and Geophysical Surveys Miscellaneous Publication 133, v. 1.2, 1 sheet, scale 1:3,000,000, http://www.dggs.dnr.state.ak.us/pubs/ pubs? reqtype $=$ citation $\& I D=20181$.

Smith, W.H.F., and Sandwell, D.T., 1997, Global seafloor topography from satellite altimetry and ship depth soundings: Science, v. 277, p. 1957-1962. 


\section{Glossary of Selected Terms and Acronyms}

AAWU "Alaska Aviation Weather Unit" of the National Weather Service.

'a`a Hawaiian term for lava flows characterized by a rough, jagged, blocky surface.

AEIC Alaska Earthquake Information Center.

AFTN Aeronautical Fixed Telecommunications Network.

AKDGGS Alaska Division of Geological \& Geophysical Surveys.

AKDT "Alaska Daylight Time"; UTC -8 hours AKDT.

AKST "Alaska Standard Time"; UTC -9 hours AKST.

andesite volcanic rock composed of about 53-63 percent silica ( $\mathrm{SiO} 2$,; an essential constituent of most minerals found in rocks).

ash fine fragments (less than 2 millimeters across) of lava or rock formed in an explosive volcanic eruption.

ASL above sea level.

ASTER Advanced Spaceborne Thermal Emission and Reflection Radiometer.

Aura a multi-national NASA scientific research satellite in orbit around the Earth, studying the Earth's ozone layer, air quality and climate.

AVHRR "Advanced Very High Resolution Radiometer"; AVHRR provides one form of satellite imagery.

AVO Alaska Volcano Observatory.

basalt general term for dark-colored igneous rock, usually extrusive, containing about 45-52 weight percent silica ( $\mathrm{SiO} 2$, an essential constituent of most minerals found in rocks).

bomb boulder-size chunk of partly solidified lava explosively ejected from a volcano.

BTD Brightness temperature difference. The subtraction of channel 5 data from channel 4 is BTD. caldera a large, roughly circular depression usually caused by volcanic collapse or explosion.

CAVW Smithsonian Institute's "Catalog of Active Volcanoes of the World" (Simkin and Siebert, 1994).

cinder cone small, steep-sided conical hill built mainly of cinder, spatter, and volcanic bombs.

COSPEC "Correlation Spectrometer," a device for measuring sulfur-dioxide emissions.

CWSU "Center Weather Service Unit" of the National Oceanic and Atmospheric Administration, stationed at the Air Route Traffic Control Center.

CV0 Cascade Volcano Observatory.

DFD German Remote Sensing Data Center.

DLR German Aerospace Center.

ERSDAC Earth Remote Sensing Data Analysis Center.

FAA Federal Aviation Administration.

fallout a general term for debris which falls to the Earth from an eruption cloud.

fault A fracture along which the blocks of the Earth's crust on either side have moved relative to one another parallel to the fracture.

FIR Flight Information Region.

fissure a roughly linear or sinuous crack or opening on a volcano; a type of vent which commonly produces lava fountains and flows.

FLIR "Forward Looking Infrared Radiometer," used to delineate objects of different temperature.

fumarole a small opening or vent from which hot gases are emitted.

GINA Geographic Information Network of Alaska. GINA is a mechanism within the University of Alaska for sharing data and technical capacity among Alaskan, Arctic, and world communities. 
GSFC Goddard Space Flight Center.

glaciolacustrine pertaining to sediments deposited in glacial lakes, and resulting landforms.

GMS Geostationary Meteorological Satellite.

GOES Geostationary Operational Environmental Satellite.

GPS Global Positioning System.

GVN "Global Volcanism Network" of the Smithsonian Institution.

Holocene geologic epoch extending from the present to 10,000 years ago.

incandescent glowing red or orange due to high temperature.

InSAR Interferometric Synthetic Aperture Radar.

interferogram a pattern formed by wave interference, especially one represented in a photograph or diagram

intracaldera refers to something within the caldera.

ISS International Space Station.

JAROS Japan ASTER Science Team.

JMA Japanese Meteorological Agency.

JPEG “Joint Photographic Experts Group," type of digital photographic file.

Ka thousands of years before the present.

lapilli pyroclasts or volcanic fragments that are between $2 \mathrm{~mm}$ and $64 \mathrm{~mm}$ in diameter.

lava molten material at the Earth's surface.

magma molten material below the surface of the Earth.

METI Ministry of Economy, Trade, and Industry.

MODIS Satellite-based "Moderate-resolution Imaging Spectroradiometer."

MWO Meteorological Watch Office.

NASA National Aeronautics and Space Administration.

NESDIS NOAA's National Environmental Satellite, Data, and Information Service.

NOAA National Oceanic and Atmospheric Administration.

NOGAPS Navy Operational Global Atmospheric Prediction System.

NOPAC North Pacific air route corridors.
NOTAM "Notice to Airmen," a notice containing information [not known sufficiently in advance to publicize by other means] concerning the establishment, condition, or change in any component [facility, service, or procedure of, or hazard in the National Airspace System] the timely knowledge of which is essential to personnel concerned with flight operations.

NPS National Park Service.

NWS National Weather Service.

OMI Ozone Mapping Instrument.

phreatic activity an explosive eruption caused by the sudden heating of ground water as it comes in contact with hot volcanic rock or magma.

phreatic ash fine fragments of volcanic rock expelled during phreatic activity; this ash usually is derived from existing rock and not from new magma.

PIREP "Pilot Weather Report"; a report of meteorological phenomena encountered by aircraft in flight.

pixel contraction of "picture element." A pixel is one of the many discrete rectangular elements that form a digital image or picture on a computer monitor or stored in memory. In a satellite image, resolution describes the size of a pixel in relation to area covered on the ground. More pixels per unit area on the ground means a higher resolution.

PK "Petropavlovsk"; capital city of Kamchatka, Russia.

Pleistocene geologic epoch extending from 2-3 million years ago to approximately 10,000 years before present.

PUFF a volcanic ash tracking model (see at URL: http://puff.images.alaska.edu/ monitoring.shtml).

pyroclast an individual particle ejected during a volcanic eruption; usually classified by size, for example, ash, lapilli.

RSAM Real-time Seismic-Amplitude Measurement.

regional earthquake earthquake generated by fracture or slippage along a fault; not caused by volcanic activity.

RFE Russian Far East.

SAB "Synoptic Analysis Branch" of NOAA.

SAR Synthetic Aperture Radar. 
satellite cone a subsidiary volcanic vent located on the flank of a larger volcano.

seismic swarm a flurry of closely spaced earthquakes or other ground shaking activity; often precedes an eruption.

shield volcano a broad, gently sloping volcano usually composed of fluid lava flows of basalt composition (for example, Mauna Loa, Hawaii).

SI International System of Units.

SIGMET SIGnificant METeorological information statement, issued by NWS.

SRTM Shuttle Radar Topography Mission.

Stratovolcano: Also called a stratocone or composite cone, a steep-sided volcano, usually conical in shape, built of interbedded lava flows and fragmental deposits from explosive eruptions.

Strombolian type of volcanic eruption characterized by intermittent bursts of fluid lava, usually basalt, from a vent or crater as gas bubbles rise through a conduit and burst at the surface.

sub-plinian style of explosive eruptions characterized by vertical eruption columns and widespread dispersal of tephra.

SVA Suspect Volcanic Activity.

\section{SVERT "Sakhalin Volcanic Eruption} Response Team" monitors and reports on Kurile Island volcanoes.

SWIR Short Wave Infrared.

tephra a general term covering all fragmental material expelled from a volcano (ash, bombs, cinders, etc.).
TA thermal anomaly.

TerraSAR-X A German built active radar microwave $\mathrm{x}$-band imaging satellite.

TFR “Temporary Flight Restriction," issued by FAA.

TIR Thermal Infrared.

UAFGI University of Alaska Fairbanks Geophysical Institute.

USCG United States Coast Guard.

USFWS United States Fish and Wildlife

Service.

USGS United States Geological Survey.

UTC "Coordinated Universal Time"; same as Greenwich Mean Time (GMT).

UUA Urgent pilot report.

VAAC Volcanic Ash Advisory Center.

VAA Volcanic Ash Advisory.

vent an opening in the earth's surface through which magma erupts or volcanic gasses are emitted.

VNIR Very Near Infrared.

volcano-tectonic earthquakes earthquakes generated within or near a volcano from brittle rock failure resulting from strain induced by volcanic processes.

Vulcanian style of explosive eruption consisting of repeated, violent ejection of incandescent fragments of viscous lava, usually in the form of blocks, along with volcanic ash. Sometimes, Vulcanian eruptions involve water mixing with erupting magma. 



\section{Appendix 1. Volcano Alert Levels and Aviation Color Codes Used by United States Volcano Observatories.}

\section{Appendix 1. Volcano Alert Levels and Aviation Color Codes Used by United States Volcano Observatories.}

Alert levels address the overall activity at the volcano, not just the hazard to aviation. There may be situations where a volcano is producing lava flows that are dangerous on the ground and merit a WATCH or WARNING, however, the hazard to aviation is minimal. Alert levels announcements contain additional explanation of volcanic activity and expected hazards where possible. (Gardner and Guffanti, 2006).

\begin{tabular}{|c|l|}
\hline \multicolumn{2}{|c|}{ Alert Levels } \\
\hline NORMAL & $\begin{array}{l}\text { Volcano is in typical background, noneruptive state. } \\
\text { Or, after a change from a higher level: } \\
\text { Volcanic activity has ceased and volcano reverted to its noneruptive state. }\end{array}$ \\
\hline ADVISORY & $\begin{array}{l}\text { Volcano is exhibiting signs of elevated unrest above known background level. } \\
\text { Or, after a change from a higher level: } \\
\text { Volcanic activity has decreased significantly but continues to be closely monitored for possible renewed increase. }\end{array}$ \\
\hline WATCH & Volcano is exhibiting heightened or escalating unrest with increased potential of eruption, timeframe uncertain. \\
& Or: \\
& A minor eruption is underway that poses limited hazards. \\
\hline WARNING & Eruption is underway but poses limited hazards. \\
\hline
\end{tabular}

\begin{tabular}{|l|l|}
\hline \multicolumn{2}{|l}{ Level of Concern Codes for Aviation } \\
\hline GREEN & $\begin{array}{l}\text { Volcano is in typical background, noneruptive state. } \\
\text { Or, after a change from a higher level: } \\
\text { Volcanic activity has ceased and volcano has returned to noneruptive background state. }\end{array}$ \\
\hline \multirow{2}{*}{ YELLOW } & $\begin{array}{l}\text { Volcano is exhibiting signs of elevated unrest above known background level. } \\
\text { Or, after a change from a higher level: } \\
\text { Volcanic activity has decreased significantly but continues to be closely monitored for possible renewed increase. }\end{array}$ \\
\hline \multirow{2}{*}{ ORANGE } & $\begin{array}{l}\text { Volcano is exhibiting heightened or escalating unrest with increased potential of eruption, timeframe uncertain. } \\
\text { Eruption is underway with no or minor ash emissions [ash-plume height specified, if possible]. }\end{array}$ \\
\hline Eruption is imminent with significant emission of volcanic ash into the atmosphere likely. \\
Or: \\
$\begin{array}{l}\text { Eruption is underway or suspected with significant emission of volcanic ash into the atmosphere [ash-plume } \\
\text { height specified, if possible]. }\end{array}$
\end{tabular}



Publishing support provided by the U.S. Geological Survey Science Publishing Network, Tacoma Publishing Service Center

For more information concerning the research in this report, contact the Director, Volcano Science Center

U.S. Geological Survey

4230 University Drive

Anchorage, Alaska 99508

http://volcanoes.usgs.gov/ 
